\title{
An Investigation on Thermoelastic Damping of High-Q Ring Resonators
}

\author{
S.T. Hossain ${ }^{\mathrm{a}}$, S. McWilliam ${ }^{\mathrm{a}, \mathrm{b}, *}$, A.A. Popov ${ }^{\mathrm{a}}$ \\ ${ }^{a}$ Faculty of Engineering, University of Nottingham, University Park, Nottingham NG7 2RD, UK \\ ${ }^{b}$ Faculty of Engineering, University of Nottingham Malaysia Campus, Semenyih, Malaysia
}

\begin{abstract}
For applications requiring high performance angular rate measurements it is important to be able to design MEMS rate sensors with high quality factors $(Q)$. This paper considers ring resonator based rate sensors and investigates the influence of design changes to the ring and support legs on thermoelastic damping, which is the dominant dissipation mechanism. A computational method is used to quantify the thermoelastic damping and a detailed parameter study is conducted to understand the influence of ring geometry, support legs and micro-machined slots around the ring circumference. The results show that damping in the support legs can have significant influence on the total energy dissipated from the resonator, and the optimum leg geometry can be identified to achieve high $Q$. It is also observed that the addition of slots improves $Q$ for resonators having higher energy loss. However, for high-Q, rings slots have a detrimental effect. The results presented are useful for designing ring resonators with reduced levels of damping.
\end{abstract}

Keywords: thermoelastic damping, ring resonator, rate gyro, quality factor, MEMS device

\section{Introduction}

Micro Electro-Mechanical Systems (MEMS) angular rate sensors are used in many applications due to their small size, light weight and ease of fabrication, replacing the conventional gyroscopes (mechanical or optical). Most of the MEMS rate sensors are classified as vibratory gyroscopes using simple mass, discs or shells as the vibrating element of the resonator. The research presented here focuses on ring resonators which feature in some commercial devices.

Sensors capable of high accuracy rate measurements have been receiving significant attention [1. However, the device performance is affected by physical damping mechanisms which influence the overall quality factor (Q-factor or Q-) of the device. The Q-factor is a measure of energy loss from the resonator per cycle

\footnotetext{
* Corresponding author

Email address: Stewart.Mcwilliam@nottingham.ac.uk (S. McWilliam)
} 
of oscillation. High performances from a damping perspective can be achieved by identifying the dominant damping mechanism and considering different ways to reduce damping. There are several different energy dissipation mechanisms present in MEMS resonators: fluid damping [2, 3], support loss [4, 5, 6], thermoelastic damping [7, 8, 9] and surface loss [2, 10]. Fluid losses are negligible if the device is vacuum encapsulated, and surface losses are more significant in Nano-Electromechanical Systems (NEMS) devices due to increasing surface to volume ratio [11. Support loss is an important energy dissipation mechanism in the context of MEMS resonators. It is the dissipation of energy due to elastic wave propagation through the supports to the substrate of the resonator, and most published research has studied support loss in beam [4, 5] and disk [12, 13] resonators. Chouvion et al. [6] predicted a very high-Q for support loss in supported ring resonators. The author of this paper also investigated the phenomenon, and the results suggested that the support loss is negligble in ring resonators. The symmetry nature of ring and supports makes it a dynamically balanced system, which translates in to a high-Q for support loss.

Thermoelastic damping (TED) has been identified as the most important energy dissipation mechanism in many MEMS resonators such as beams [9, 14, 15] and rings [16]. It is an intrinsic source of energy dissipation which occurs due to coupling between thermal and mechanical states within the structure. The coupling induces temperature gradients by converting mechanical energy of a vibrating body to thermal energy; the energy dissipates via irreversible heat flow. Since TED is present in every structure, materials with low thermal expansion and high thermal conductivity are desired to minimize TED [17. However, the majority of MEMS devices are manufactured using silicon based materials which often limits the selection of desired material for minimizing TED. Thus, efforts to improve the performance level of sensors are normally based on selecting geometries that yield reduced damping.

A limited number of studies on ring based rate sensors have been performed in the past. Ayazi and Najafi [16] presented a detailed analysis on the design, fabrication and experimental testing of a polysilicon ring gyroscope, although the damping behaviour of the sensor was not investigated. Wong et al. [18, 19] and Hao and Ayazi [20] developed analytical models to predict thermoelastic damping in ring structures, and this work explored the effect of ring dimension on the Q-factor due to thermoelastic loss. However these models are not capable of taking into account support legs or slots. Support legs are used to attach the ring resonators to the substrate, and although the support legs are designed to have minimal impact on the natural frequencies of the ring, they flex when the ring vibrates and form an additional source of TED which influences the overall damping. Incorporating micro-machined slots in flexural beam resonators has been shown [21, 22, 23, to reduce TED. These slots affect the thermo-mechanical coupling and can have 
significant influence on the Q-factor. The impact of support legs and slots around the circumference of the ring will be considered in this study. Since the support loss is negligble in ring resonators, it has not been considered in this paper.

With the ultimate aim of realising high performance rate sensors, the purpose of this paper is to gain improved understanding of TED in ring resonators by taking into account the resonator support structure and slots. Based on the fully coupled thermo-mechanical eigen-problem (e.g. 24]), the finite element method (COMSOL 25]) is used to model and predict TED. Parameter studies are performed to investigate the effects of ring dimensions on frequency and Q-factor. In particular, the study focuses on obtaining high-Q resonators by changing the bulk geometry of the ring and the support legs, and investigating the impact of slots around the circumference of the ring. The analyses presented in this paper are thought to be useful for designing high-Q ring resonators to improve performance, from the point of view of thermoelastic damping.

The paper is organized into different sections as follows. Section 2 provides a brief overview of the principle of operation of ring based rate sensors. Section 3 reviews the background of thermoelastic damping based on Zener's classical theory. Section 4 describes the methodology used, including the basic principles of deriving coupled equations of thermoelasticity and constructing the numerical model in COMSOL. In Sections 5 to 7 , the numerical model is validated and used to investigate TED in rings, support legs and slotted rings. The damping behaviour of the resonator is discussed and analysed based on the results throughout. Finally, the main findings from the present study are outlined with concluding remarks in Section 8 .

\section{Ring Based Rate Sensors}

Ring based rate sensors measure the angular velocity of a body about the polar axis of the ring, based on Coriolis effect. For a perfect ring the modes occur in degenerate pairs of equal frequencies and the flexural $2 \theta$ in-plane modes are normally utilised to detect angular rate, see Figure 1 The operation of the sensor involves mechanically exciting the primary mode and measuring the response of the secondary mode, which is directly proportional to angular rate. In Figure 1, the secondary mode is located geometrically $45^{\circ}$ apart from the primary mode [16]. Further details about the operation of rate sensors are given in Ref. [26].

The ring resonator is supported by legs, and Figure 2 shows three different support leg configurations considered in this paper. However, the purpose of the paper is to provide an understanding of the damping behaviour in supported ring resonators irrespective of the leg design. Figure 2(a) shows support legs consisting of 8 pairs of 'z' shaped support legs connected to a circular central hub, this configuration is considered 


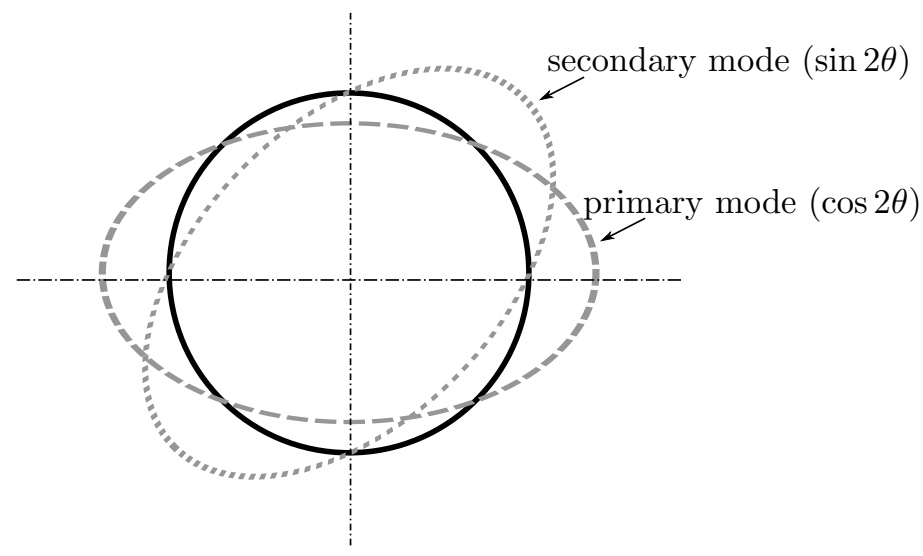

Figure 1: Degenerate pair of $2 \theta$ in-plane modes of a ring resonator. Dashed Line: primary mode (cos $2 \theta$ ) and dotted line: secondary mode $(\sin 2 \theta)$.

in most studies later. Figure 2(b) shows the support leg configuration considered in [16] and consists of 16 semi-circular support legs connected to a central hub. Figure 2(c) shows a configuration with the 8 support legs connected outside of the ring, with the "free ends" attached to a substrate. In all cases, it is important to maintain symmetry relative to the $2 \theta$ modes to ensure the natural frequencies do not split [16].

\section{Thermoelastic Damping}

Thermo-elastic Damping (TED) occurs in any structure made from a thermoelastic solid that is subjected to a cyclic stress. Zener [7, 8] was the first to develop the theory of thermoelastic damping for thin rectangular beams under flexural vibrations, and the theory has been extended to ring structures [18].

The bending of a structure during its vibration causes regions of compression and tension to develop. The region under compression is heated and the stretched tension region is cooled (see Figure 3). For a material with a non-zero thermal expansion coefficient, a temperature gradient is generated which relaxes by irreversible heat flow from hotter to cooler region producing entropy, leading to energy dissipation. In other words, mechanical energy is converted to irrecoverable thermal energy. This is known as thermoelastic relaxation and the characteristic time it takes to equalize the temperature is called the relaxation time $(\tau)$. The relaxation time is one of the key parameters in Zener's approximate expression for thermoelastic damping given by

$$
Q_{T E D}^{-1}=\Delta_{M} \frac{\omega_{0} \tau}{1+\left(\omega_{0} \tau\right)^{2}}
$$

where the effective relaxation time is $\tau=\frac{C_{v} b^{2}}{k \pi^{2}}$ and the relaxation strength is $\Delta_{M}=\frac{E \alpha^{2} T_{0}}{C_{v}}$.

Here, $Q_{T E D}$ is the Q-factor due to thermoelastic damping, $\omega_{0}$ is the fundamental undamped natural 


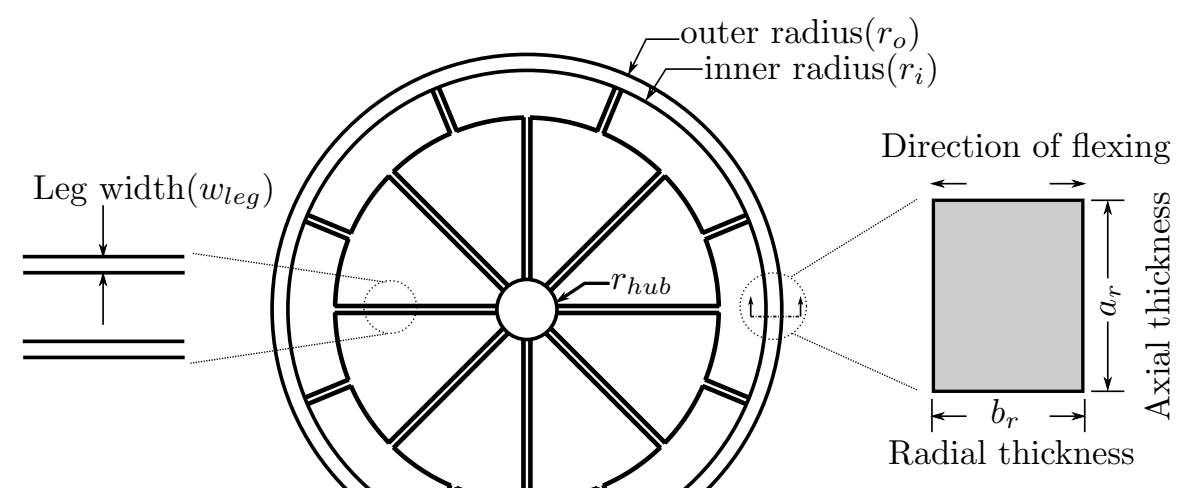

$\underline{\text { Ring cross-section }}$

(a)

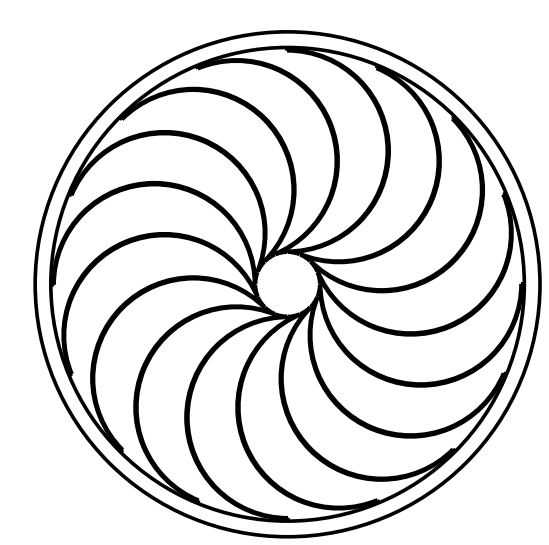

(b)

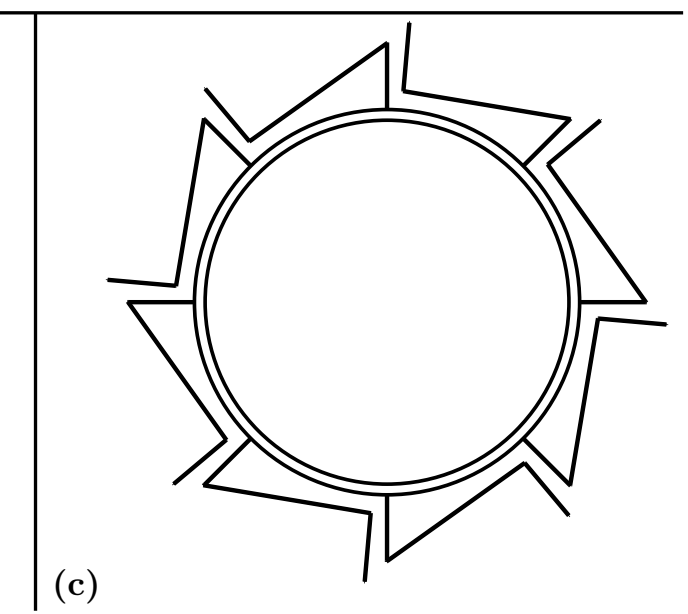

Figure 2: The ring resonator with different support leg configurations: (a) pair of 'z' shaped legs, (b) semi-circular legs, and (c) outer support legs. 


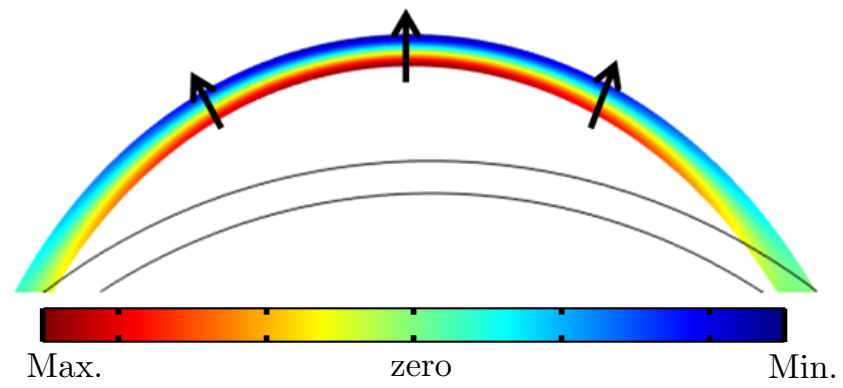

Figure 3: Temperature distribution in a ring section under flexure. Red and blue indicates higher and lower temperatures, respectively. Black arrows indicate the direction of heat transfer.

frequency, $C_{v}\left(=\rho C_{p}\right)$ is the specific heat capacity at constant volume, $T_{0}$ is the reference temperature, $\alpha$ is the linear coefficient of thermal expansion, $E$ is the Young's modulus, $k$ is the thermal conductivity, and $b$ is the dimension in the direction of the flexing. For a ring vibrating in its in-plane flexure mode, $b$ and $\omega_{0}$ are the radial thickness $\left(b_{r}\right)$ and undamped natural frequency of the ring, respectively. Based on Zener's theory there are three conditions that influence the value of $Q_{T E D}: \tau \approx \omega_{0}^{-1}\left(\omega_{0} \tau \approx 1\right), \tau \gg \omega_{0}^{-1}$ $\left(\omega_{0} \tau \gg 1\right)$ and $\tau \ll \omega_{0}^{-1}\left(\omega_{0} \tau \ll 1\right)$. When $\tau \ll \omega_{0}^{-1}$, i.e. the low frequency range, the vibration is considered isothermal and a very small amount of energy dissipates from the solid. At this low frequency, only a small temperature gradient is formed. This is due to the fact that the temperature gradient is dependent on strain rate. In the low frequency range, the strain rate is reduced, consequently the temperature gradient reduces, leading to small levels of energy dissipation. In the high frequency range, i.e. $\tau \gg \omega_{0}^{-1}$, very small levels of energy is dissipated, similar to low frequency range and the system is adiabatic. Due to the high frequency, the temperature gradient has a shorter time period to relax back to the equilibrium state, leading to very low energy dissipation. During this shorter time period the temperature gradient vanishes before heat transport occurs. When $\tau \approx \omega_{0}^{-1}$, maximum energy dissipation occurs, i.e. $Q_{T E D}$ is at a minimum. In this case, the relaxation time and flexural period of the system are of the same order of magnitude, allowing the temperature gradient to relax, through heat transport, to restore thermal equilibrium. This maximum dissipation occurs at the Debye peak (Figure 4 ) and is defined by $Q_{T E D}=Q_{\min }=\frac{2}{\Delta_{M}}$. From the thermodynamic point of view, the thermal energy, which is considered lost, increases the entropy of the system. Although Zener's closed form expression provides an accurate approximation of $Q_{T E D}$ for simple beam and ring-like structures, it is based on some simplifying assumptions. One of the important assumptions is that heat conduction only occurs in the direction of flexing and neglects heat conduction along the longitudinal axis. Zener also showed that for a beam vibrating in its transverse mode, 98.6\% of the relaxation occurs within the first transverse thermal mode [9] and hence, a single relaxation time is 


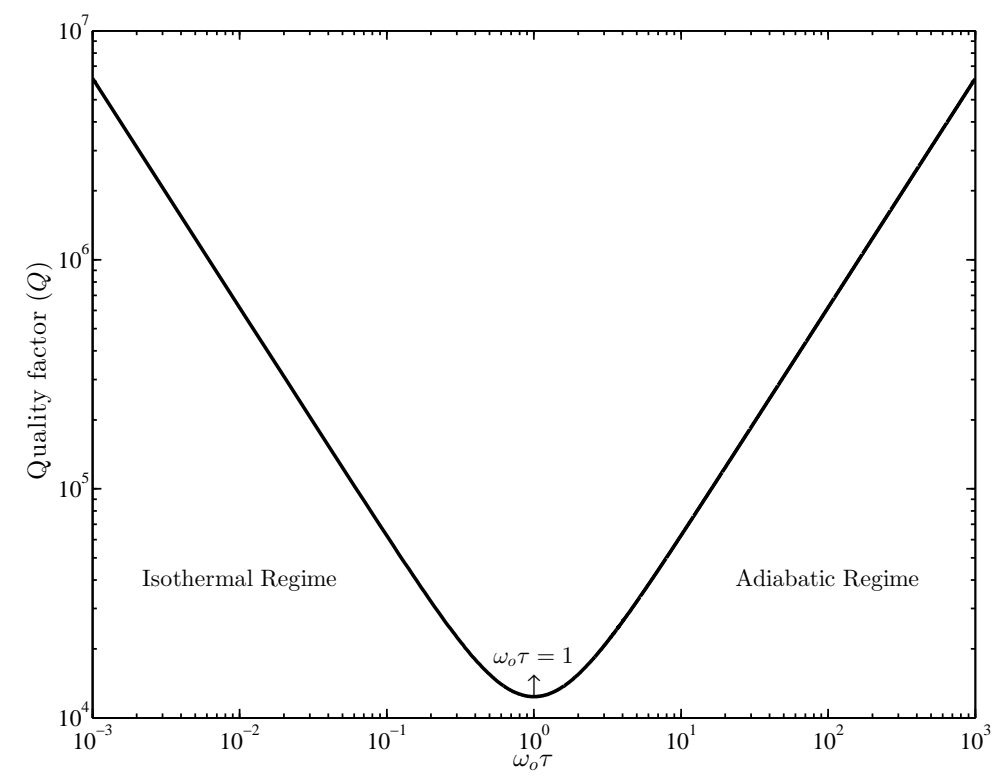

Figure 4: $Q_{T E D}$ as a function of resonant frequency. The Debye peak is observed when $\tau \approx \omega_{0}^{-1}$.

considered for the calculation of $Q_{T E D}$. This assumption induces a very small error in the prediction of $Q_{T E D}$

Kinra and Milligan [27] and Lifshitz and Roukes (L-R) 9] developed exact, closed-form expressions for TED in this case. L-R's solution technique is widely used to estimate TED in micro- and nano-scale resonators. Lifshitz and Roukes developed the following expressions for a beam in flexure [9]:

$$
Q_{T E D}=\frac{E \alpha^{2} T_{0}}{C_{v}}\left(\frac{6}{\xi^{2}} \frac{6}{\xi^{3}} \frac{\sin \xi+\sinh \xi}{\cos \xi+\cosh \xi}\right), \text { with } \xi=b \sqrt{\frac{\omega_{0}}{2 \chi}}
$$

where $b$ is again the dimension across the beam in the direction of the flexing and $\chi$ is the thermal diffusivity of the material.

Wong et al. [19] and Hao and Ayazi 20] derived expressions for $Q_{T E D}$ in a ring resonator vibrating in its in-plane flexural modes based on L-R's approach, where $b$ and $\omega_{0}$ in Eqn. (2) are the radial thickness $\left(b_{r}\right)$ and the undamped natural frequency of ring, respectively.

The aforementioned analytical expressions for TED are based on some restrictive assumptions which limit their applicability to simple structures like beams, rings, etc., vibrating in their flexural modes. Since analytical expressions are not suitable for complex geometries, e.g. ring with supporting legs, to evaluate TED, a numerical approach based on the finite element (FE) method is used in this paper. A brief overview of the FE methodology is presented in the following section. The simplified analytical results presented in 
this section are used for validation purposes later.

\section{Methodology}

\subsection{Governing Equations of Thermoelasticity}

In every solid there exists a fundamental thermoelastic coupling between the thermal and mechanical states (e.g. between stress and temperature fields), where the thermal expansion coefficient acts as the coupling constant [28. The governing equations of thermoelasticity are derived from the equations of motion of solids and a heat diffusion equation.

The equations of motion for a three dimensional (3D) elastic solid (neglecting external body force) in a Cartesian coordinate system are given by 29

$$
\begin{aligned}
& \frac{\partial \sigma_{x x}}{\partial x}+\frac{\partial \sigma_{x y}}{\partial y}+\frac{\partial \sigma_{z x}}{\partial z}=\rho \frac{\partial^{2} u}{\partial t^{2}} \\
& \frac{\partial \sigma_{x y}}{\partial x}+\frac{\partial \sigma_{y y}}{\partial y}+\frac{\partial \sigma_{y z}}{\partial z}=\rho \frac{\partial^{2} v}{\partial t^{2}} \\
& \frac{\partial \sigma_{z x}}{\partial x}+\frac{\partial \sigma_{y z}}{\partial y}+\frac{\partial \sigma_{z z}}{\partial z}=\rho \frac{\partial^{2} w}{\partial t^{2}}
\end{aligned}
$$

where $u, v, w$ are displacements, and $\frac{\partial^{2} u}{\partial t^{2}}, \frac{\partial^{2} v}{\partial t^{2}}, \frac{\partial^{2} w}{\partial t^{2}}$ are the acceleration of an infinitesimal element of the body, in the $x, y$ and $z$ directions. $\rho$ is the density of the material.

The constitutive equations of thermoelasticity for a homogeneous isotropic material is given by [29]

$$
\sigma_{i j}=2 \mu \varepsilon_{i j}+(\Lambda e-\beta T) \delta_{i j}
$$

where $\sigma_{i j}$ and $\varepsilon_{i j}$ are the stress and strain tensors $(i, j=x, y, z)$, respectively. The thermoelastic constant, $\beta=\alpha(3 \Lambda+2 \mu)$ and the strain dilatation $e=\varepsilon_{x x}+\varepsilon_{y y}+\varepsilon_{z z}$. Also, $T$ is the temperature change from the reference temperature $\left(T_{0}\right)$ and $\alpha$ is the thermal expansion coefficient. Here, one has the Lamé constants, $\Lambda=\frac{E}{(1+\nu)(1-2 \nu)}$ and $\mu=\frac{E}{2(1+\nu)} \cdot \nu$ is the Poisson's ratio of the material. The Kronecker delta is defined by

$$
\delta_{i j}=\left\{\begin{array}{rr}
1 & \text { when } i=j \\
0 & \text { when } i \neq j
\end{array}\right.
$$

The combination of Eqs. (3) to (6), and linear strain displacement equations give the following set of 
equations 24.

$$
\begin{aligned}
& \rho \frac{\partial^{2} u}{\partial t^{2}}=\mu\left(\frac{\partial^{2} u}{\partial x^{2}}+\frac{\partial^{2} u}{\partial y^{2}}+\frac{\partial^{2} u}{\partial z^{2}}\right)+(\Lambda+\mu)\left(\frac{\partial^{2} u}{\partial x^{2}}+\frac{\partial^{2} v}{\partial x \partial y}+\frac{\partial^{2} w}{\partial x \partial z}\right)-\beta \frac{\partial T}{\partial x} \\
& \rho \frac{\partial^{2} v}{\partial t^{2}}=\mu\left(\frac{\partial^{2} v}{\partial x^{2}}+\frac{\partial^{2} v}{\partial y^{2}}+\frac{\partial^{2} v}{\partial z^{2}}\right)+(\Lambda+\mu)\left(\frac{\partial^{2} u}{\partial y \partial x}+\frac{\partial^{2} v}{\partial y^{2}}+\frac{\partial^{2} w}{\partial y \partial z}\right)-\beta \frac{\partial T}{\partial y} \\
& \rho \frac{\partial^{2} w}{\partial t^{2}}=\mu\left(\frac{\partial^{2} w}{\partial x^{2}}+\frac{\partial^{2} w}{\partial y^{2}}+\frac{\partial^{2} w}{\partial z^{2}}\right)+(\Lambda+\mu)\left(\frac{\partial^{2} u}{\partial z \partial x}+\frac{\partial^{2} v}{\partial z \partial y}+\frac{\partial^{2} w}{\partial z^{2}}\right)-\beta \frac{\partial T}{\partial z}
\end{aligned}
$$

The heat conduction equation in 3D for thermoelastic damping is given by 24]

$$
\rho C_{p} \frac{\partial T}{\partial t}=k\left(\frac{\partial^{2} T}{\partial x^{2}}+\frac{\partial^{2} T}{\partial y^{2}}+\frac{\partial^{2} T}{\partial z^{2}}\right)-T_{0} \beta \frac{\partial e}{\partial t}
$$

where $C_{p}$ is the specific heat at constant pressure.

In MEMS, the temperature fluctuations from the equilibrium temperature are considered small, that is why Eq. 10 has been linearised about the reference temperature, $T_{0}$.

Eqs. (7) to (10) are known as the coupled equations of thermoelasticity. The coupling between structural Eqs. (7) to (9) with the thermal Eq. (10) is via the thermal expansion coefficient $(\alpha)$ in the thermoelastic constant $(\beta)$. The third term on the right hand side of Eqs. (7) to (9) corresponds to thermal expansion and the second term on the right of Eq. (10) represents strain induced heat. These equations can be usefully solved using the finite element (FE) method.

\subsection{Numerical Simulation}

Eqs. (3) to (5) are integrated into the commercial finite element package COMSOL [25]. Coupling with the heat diffusion equation thermal expansion is implemented to these equations based on the constitutive relation (Eq. 6). This gives a set of equations similar to Eqs. (7) to (9). The heat source term of the diffusion Eq. 10 is specified in the heat conduction equation available in the software for the bi-directional coupling with Eqs. (7) to (9). It is considered that the vibration takes place in a vacuum so there will be no convective heat transfer with the surroundings and radiation affects are considered negligible. The adiabatic (thermal insulation) boundary condition is applied at all the external boundaries within the model.

The models are meshed using quadratic triangular elements generated by the built-in meshing technique in COMSOL. Depending on the ring and support dimensions, the total number of elements used in later 
simulations varies between $\sim 70000$ and $\sim 160000$.

The simulations are performed using two-dimensional fully coupled thermo-mechanical damped eigenfrequency analysis. The two-dimensional plane stress assumption $\left(\sigma_{z z}=\sigma_{z x}=\sigma_{z y}=0\right)$ is used to reduce the computational effort. The damped eigenfrequency analysis results in complex eigenvalues $(\lambda)$ from which the damped natural frequencies and Q-factors are evaluated.

For a lightly damped resonating system,

$$
\lambda=-\omega_{0} \zeta \pm i \omega_{0} \sqrt{1-\zeta^{2}}
$$

where $\zeta$ is damping ratio ( $\ll 1$ for light damping) and $\omega_{0}$ is the undamped natural frequency of the resonating mode. Eq. 111) can be simplified further as,

$$
\lambda=-\omega_{0} \zeta \pm i \omega_{0} \approx-\frac{\omega_{0}}{2 Q} \pm i \omega_{0}
$$

Here, the damped natural frequency, $\omega$ and quality factor, $Q$ are given by

$$
\begin{array}{r}
\omega=|\operatorname{Im}(\lambda)| \approx \omega_{0} \\
Q=\frac{1}{2} \frac{|\operatorname{Im}(\lambda)|}{|\operatorname{Re}(\lambda)|}
\end{array}
$$

Ring resonators are generally manufactured using (111)-oriented single crystal silicon due to the uniform and homogeneous material properties over the (111) surface [30. The mechanical and thermal properties of (111) silicon, used in the simulations, are summarised in Table 1

\begin{tabular}{cc} 
Table 1: Mechanical and thermal properties of silicon for ring resonator \\
\hline Young's Modulus, $E(\mathrm{GPa})$ & 170 \\
Coefficient of Thermal Expansion, $\alpha(1 / \mathrm{K})$ & $2.6 \times 10^{-6}$ \\
Thermal Conductivity, $k(\mathrm{~W} / \mathrm{m} . \mathrm{K})$ & 130 \\
Specific Heat, $C_{P}(\mathrm{~J} / \mathrm{kg} \cdot \mathrm{K})$ & 700 \\
Density, $\rho\left(\mathrm{kg} / \mathrm{m}^{3}\right)$ & 2330 \\
Poisson's Ratio, $\nu$ & 0.22 \\
Reference Temperature, $T_{0}(\mathrm{~K})$ & 300 \\
\hline
\end{tabular}




\section{Thermoelastic Damping in Unsupported Rings}

\subsection{Validation}

The purpose of this section is to validate the model developed in the previous section and provide analytical results for the natural frequency by considering the natural frequency and TED Q-factor for the flexural $2 \theta$ in-plane modes of an unsupported perfect ring. Whilst validating the model, a mesh convergence study was conducted, but for brevity, this is not included in the paper. It is well known that the natural (undamped) frequency $\left(\omega_{0}\right)$ of a ring is given by [31]:

$$
\omega_{0}=\frac{n\left(n^{2}-1\right)}{r^{2} \sqrt{n^{2}+1}} \sqrt{\frac{E I_{z}}{\rho A}}
$$

where $n$ is the mode number (for $2 \theta$ in-plane modes, $n=2$ ), $r$ is the radius of the ring, $I_{z}$ is the area moment of inertia about axial direction, $A$ is the cross-sectional area of the ring, and $\rho$ is the material density.

The natural frequency and Q-factor presented in Table 2 are for a ring resonator of 3 mm radius and $120 \mu \mathrm{m}$ radial thickness. Table 2 compares the results obtained using numerical simulation against analytical and experimental values obtained using Eqs. (1), 22, (15) and Ref. [18. The $2 \theta$ in-plane mode shape and the temperature distribution due to TED, from COMSOL, is shown in Figure 5.

Table 2: Results of a simulation for a ring vibrating in its in-plane $2 \theta$ mode

\begin{tabular}{|c|c|c|c|c|c|}
\hline \multicolumn{2}{|c|}{ Natural Frequency $(\mathrm{kHz})$} & \multicolumn{3}{|c|}{$Q_{T E D}$} & \multirow{2}{*}{ Overall, Q (Exp.[18]) } \\
\hline Analytical & Simulated & $\begin{array}{c}\text { Analytical (Zener) } \\
\text { Eq. (1) }\end{array}$ & $\begin{array}{c}\text { Analytical (L-R) } \\
\text { Eq. (2) }\end{array}$ & Simulated & \\
\hline 14.04 & 14.03 & 10569 & 10670 & 10672 & 10500 \\
\hline
\end{tabular}

Comparing the natural frequencies, it can be seen that they are in good agreement and the analytical and simulated values are almost identical. The analytical frequency is calculated based on the expression for the natural frequency of an undamped ring vibrating in its flexural $2 \theta$ (in-plane) mode. In contrast the simulated value is the damped natural frequency, so a small difference would be expected. The simulated $Q_{T E D}$ is in excellent agreement with the analytical and experimental values, with $<1 \%$ difference between the simulated and analytical values. Given that the analytical results for TED do not account for TED in the support legs or any other sources of damping, it can be deduced from the experimental result that for this case TED in the ring is the dominant damping mechanism and the influence of the support is negligible. 


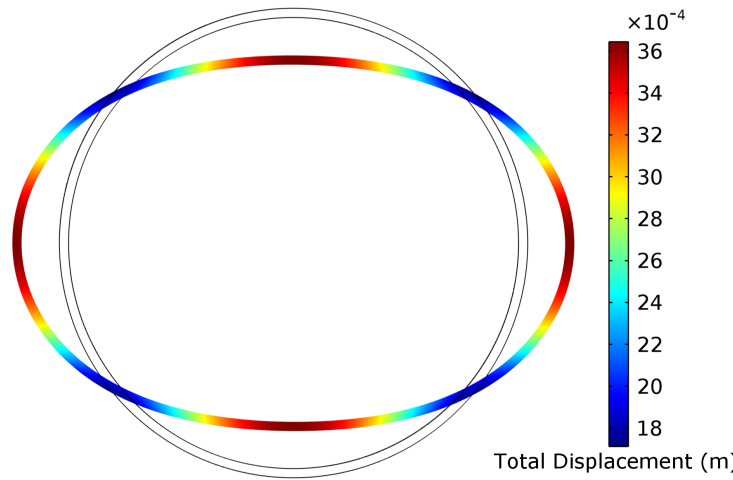

(a)

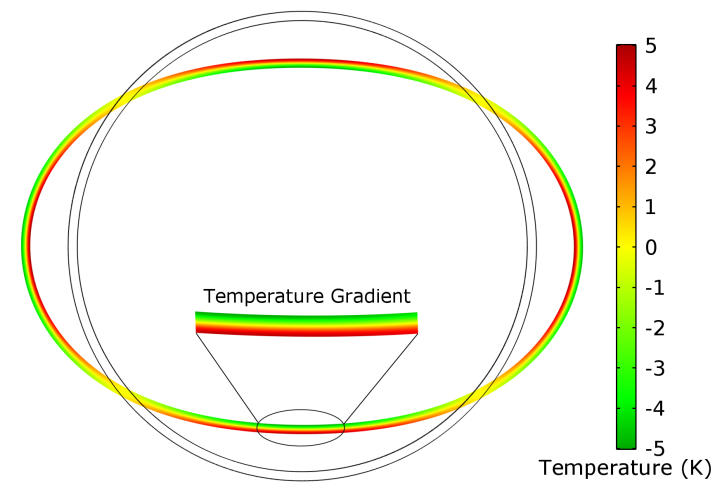

(b)

Figure 5: (a) $2 \theta$ in-plane mode shape, and (b) Temperature distribution due to thermoelastic damping in a ring of $3 \mathrm{~mm}$ radius and $120 \mu \mathrm{m}$ radial thickness.

\subsection{Parameter Study of Unsupported Rings}

The purpose of this section is to present a parameter study for the influence of ring geometry on the natural frequencies and $Q_{T E D}$ for the flexural $2 \theta$ in-plane modes of an unsupported ring.

Figures 6 and 7 show how the damped natural frequency and $Q_{T E D}$ vary as the diameter $(d)$ and radial thickness $\left(b_{r}\right)$ are varied. These results have been obtained using the finite element model, but similar results are obtained using Eqs. (1), (2) and (15).

From Eq. [15, if the density $\rho$ and Young's modulus $E$ are constant, the undamped natural frequency varies as follows:

$$
\begin{gathered}
\omega_{0} \propto \frac{1}{r^{2}} ; \text { for fixed } b_{r} \\
\omega_{0} \propto b_{r} ; \text { for fixed } r
\end{gathered}
$$

These relationships can be observed in Figure 6, and indicate that high frequencies occur for small diameter thick rings, and low frequencies occur for large diameter thin rings, as expected.

In Figure 7, it can be seen that for a given fixed diameter, the $Q_{T E D}$ decreases as $b_{r}$ decreases until it reaches a minimum value before it starts increasing again. The critical value $b_{r}$ when the minimum $Q_{T E D}$ occurs is different for each diameter. For example, for $d=8 \mathrm{~mm}$ the critical value for $b_{r}$ is $120 \mu \mathrm{m}$, whilst for $d=3 \mathrm{~mm}$ the critical value is $60 \mu \mathrm{m}$. For large values of $b_{r}, Q_{T E D}$ increases as $d$ increases. In contrast, for small values of $b_{r}, Q_{T E D}$ decreases as $d$ decreases. This study suggests that high $Q_{T E D}$ values can be achieved either with large diameter thin rings or with small diameter thick rings.

Although high $Q_{T E D}$ values can be obtained using small diameter thick rings, these configurations are 


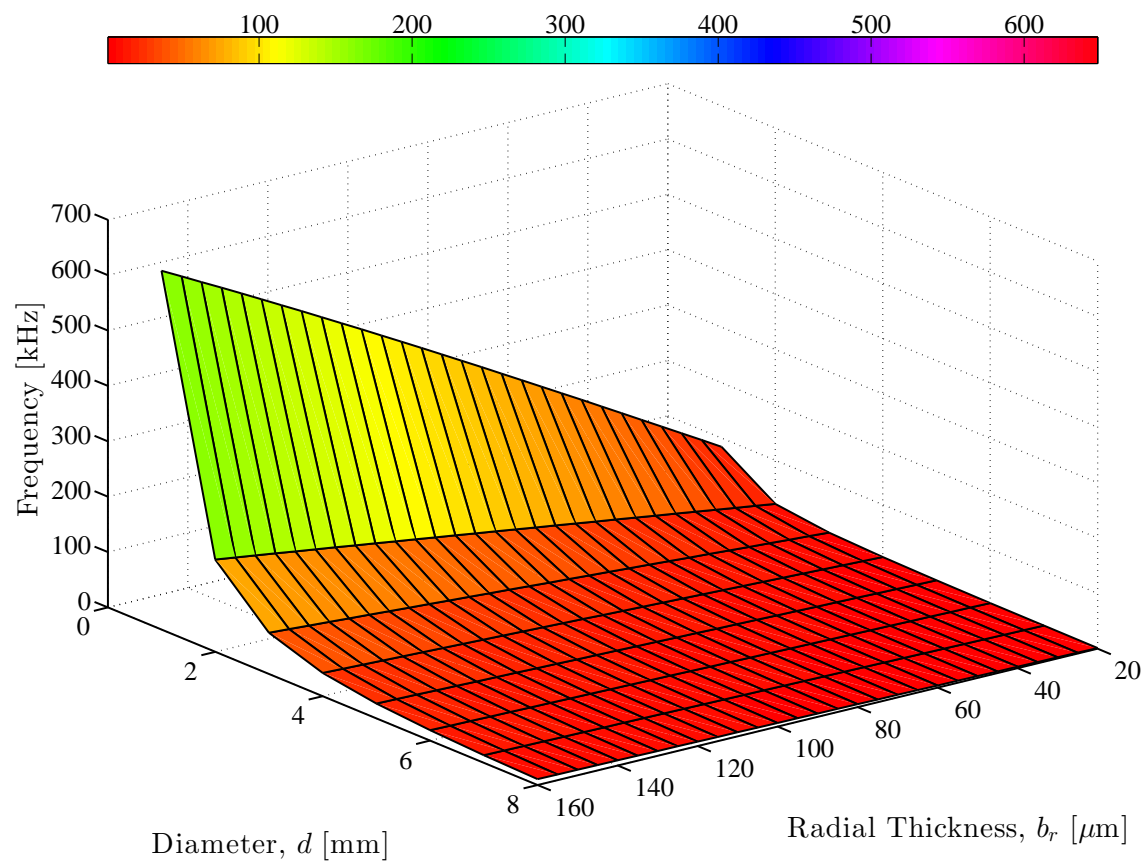

Figure 6: Frequency (damped) as a function of diameter and radial thickness.

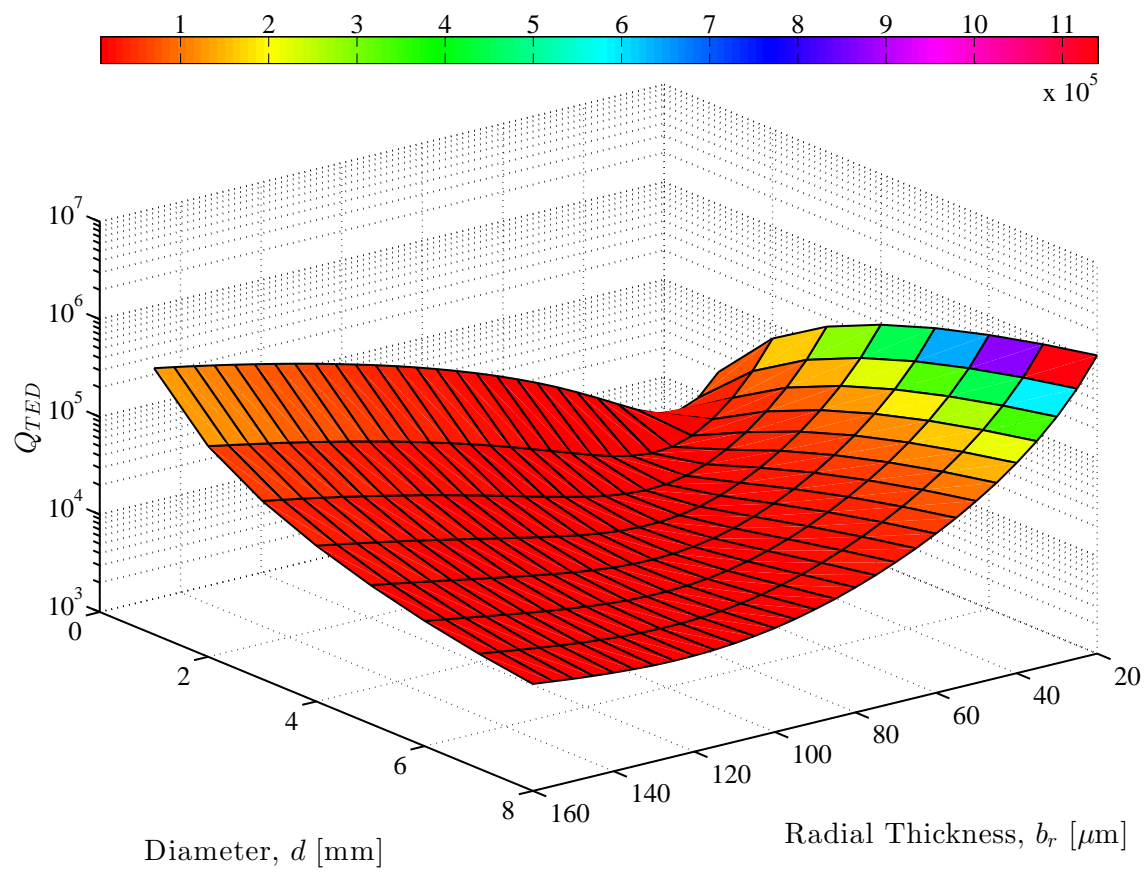

Figure 7: $Q_{T E D}$ as a function of diameter and radial thickness. 


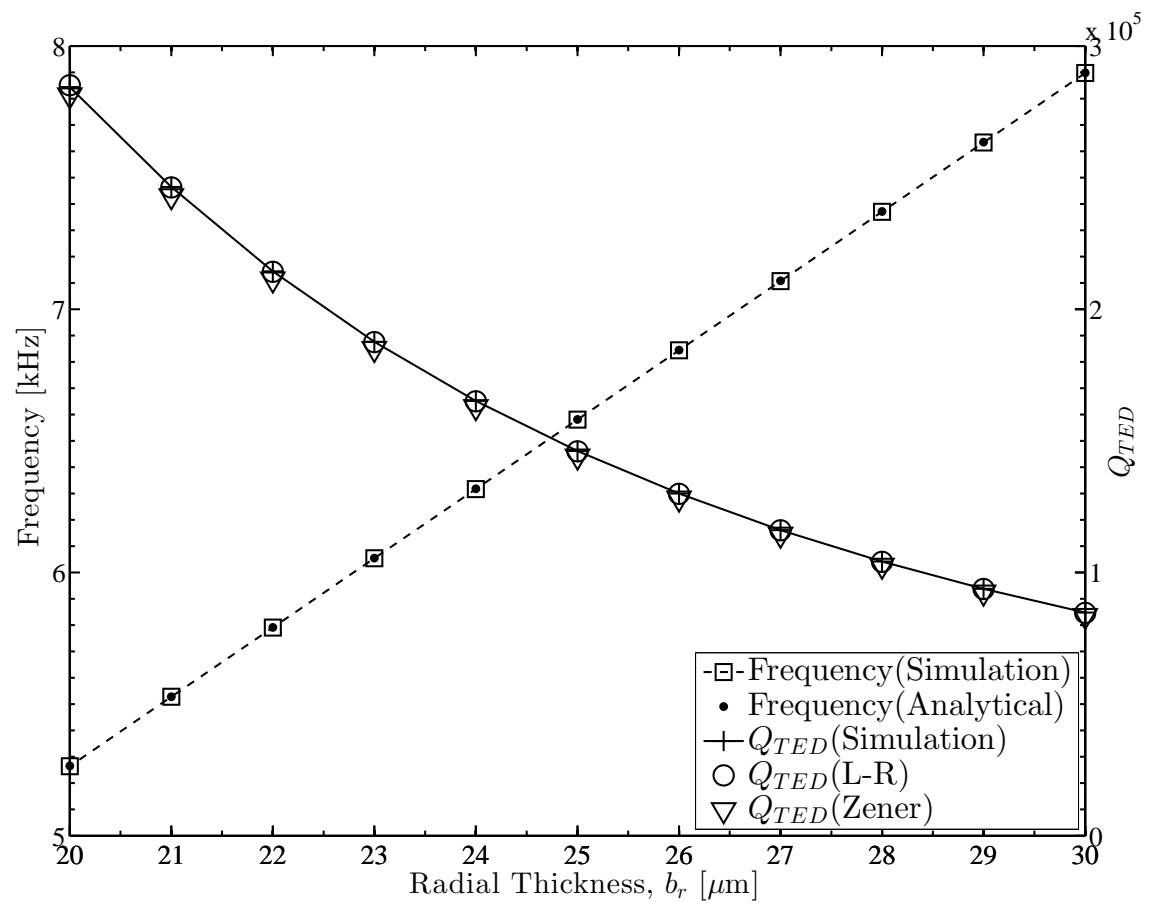

Figure 8: Frequency (damped) and $Q_{T E D}$ as a function of radial thickness for $4 \mathrm{~mm}$ diameter ring.

not suitable for practical MEMS rate sensor applications because higher frequency applications are preferred to avoid unwanted couplings and typically the frequency range is 10 to $30(\mathrm{kHz})[32$, based on published values. For this reason a trade-off is required between $Q_{T E D}$ and frequency when selecting the basic ring dimensions to achieve high $Q$. The general approach would be to consider a fixed diameter ring and then perform a parametric analysis to decide the radial thickness. In this paper, two different ring diameters $(d=4$ and $8 \mathrm{~mm})$ are considered for the purpose of comparisons.

To highlight the trade-off between frequency and Q-factor, Figure 8 shows the frequency and $Q_{T E D}$ plotted as a function of radial thickness for $d=4 \mathrm{~mm}$. The plot includes results obtained using numerical simulations as well as analytical results, and they are all in excellent agreement. It is evident that the numerically simulated $Q_{T E D}$ values are in excellent agreement with those calculated using Eq. (2). The $Q_{T E D}$ values predicted using Zener's expressions are slightly underestimated, and this is expected because Lifshitz and Roukes derived an exact expression compared to Zener's approximate expression. Similar trends in analytical and simulated results can be observed for $d=8 \mathrm{~mm}$, which are not presented here. 


\subsection{Damping Behaviour in Unsupported Rings}

In the previous section it was shown that high $Q_{T E D}$ values can be achieved either with large diameter thin rings or with small diameter thick rings, and low $Q_{T E D}$ values are obtained for critical $b_{r}$ thickness values.

To understand this behaviour, three different cases of high and low $Q_{T E D}$ are considered and these are: (a) $2 \mathrm{~mm}$ diameter ring with $160 \mu \mathrm{m}$ radial thickness, (b) $4 \mathrm{~mm}$ diameter ring with $20 \mu \mathrm{m}$ radial thickness and (c) $8 \mathrm{~mm}$ diameter ring with $120 \mu \mathrm{m}$ radial thickness. From numerical simulation, the $2 \mathrm{~mm}$ ring has a frequency $\omega$ of $166.758 \mathrm{kHz}$ and $Q_{T E D}=1.44 \times 10^{5}$, whereas the $4 \mathrm{~mm}$ ring has $\omega=5.264 \mathrm{kHz}$ and $Q_{T E D}=2.85 \times 10^{5}$ and, the $8 \mathrm{~mm}$ ring has $\omega=7.895 \mathrm{kHz}$ and $Q_{T E D}=9624$. Figure 9 shows the $Q_{T E D}$ for these rings on the Debye peak curve, and are obtained using Eqs. (1) and (15). The horizontal and vertical dashed lines show the damped natural frequency and corresponding $Q_{T E D}$ values for the three rings considered. It is evident that the $4 \mathrm{~mm}-20 \mu \mathrm{m}$ ring operates in the isothermal region, i.e. small temperature gradient is formed during its vibration, and this leads to very low energy dissipation, high $Q_{T E D}$. In contrast, the $8 \mathrm{~mm}-120 \mu \mathrm{m}$ ring operates in the region very close to the Debye peak where maximum dissipation occurs. In this case, the flexural period and relaxation time have the same order of magnitude, resulting in a low $Q_{T E D}$ value. However, the $2 \mathrm{~mm}-160 \mu \mathrm{m}$ ring operates in the adiabatic region due to its high frequency. Since the thermal gradient vanishes due to the shorter time period, it exhibits a high $Q_{T E D}$ value. In summary, the small diameter thick rings and large diameter thin rings have high $Q_{T E D}$ due to operating in the adiabatic and isothermal regions, respectively. For thicker rings, one should be careful about the practical limitations that exist for ring geometries, i.e. $b_{r}<\frac{d}{2}$. This ensures that the geometry is not a disk/plate.

\section{Thermoelastic Damping in Supported Rings}

\subsection{Effect of Support Legs on the Frequency and $Q_{T E D}$}

To investigate the effect of the support legs on frequency and $Q_{T E D}, 4 \mathrm{~mm}$ diameter rings with radial thicknesses in the range $20-25 \mu \mathrm{m}$ are simulated with support legs $\left(w_{l e g}=6 \mu \mathrm{m}\right)$ connected to the resonator. As was seen in Figure 7 this range of $b_{r}$ values gives high $Q_{T E D}$ for unsupported rings. In this study, the leg configuration shown in Figure 2(a) is used to support the ring. Table 3 summarises the numerically simulated results for frequency and $Q_{T E D}$ and compares results for supported and unsupported rings. The results suggest both the frequency and $Q_{T E D}$ increase when the support is added to the ring structure. Although the support legs provide additional modal mass and stiffness to the ring, the frequency increase 


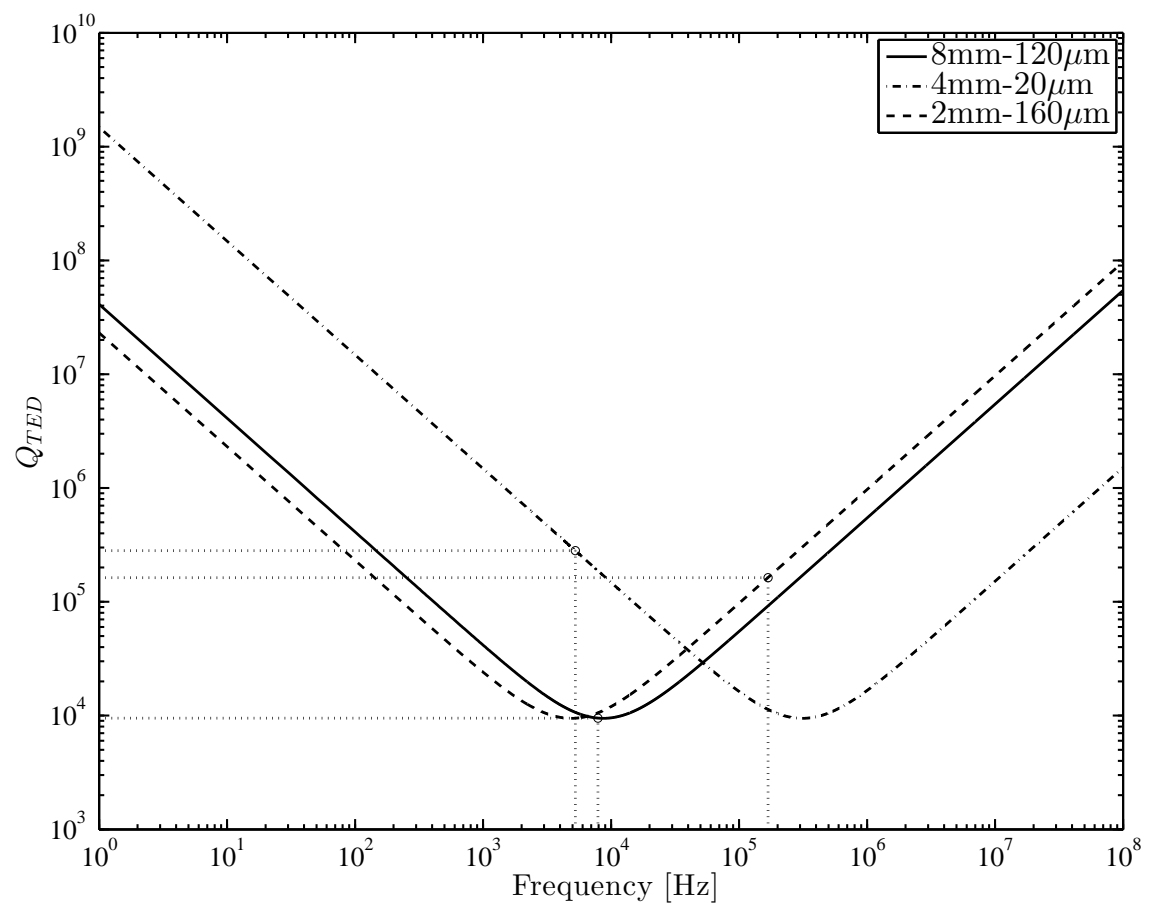

Figure 9: Debye peak curve for $2 \mathrm{~mm}-160 \mu \mathrm{m}, 4 \mathrm{~mm}-20 \mu \mathrm{m}$ and $8 \mathrm{~mm}-120 \mu \mathrm{m}$ rings.

Table 3: Frequency and Q-factor of a $4 \mathrm{~mm}$ diameter rings with and without support legs and hub

\begin{tabular}{ccccc}
\hline \multirow{2}{*}{ Radial Thickness, $b_{r}(\mu \mathrm{m})$} & \multicolumn{2}{c}{ Unsupported Ring } & \multicolumn{2}{c}{ Supported Ring } \\
\cline { 2 - 5 } & Frequency $(\mathrm{kHz})$ & $Q_{T E D}$ & Frequency $(\mathrm{kHz})$ & $Q_{T E D}$ \\
\hline 20 & 5.264 & $2.850 \times 10^{5}$ & $6.111(13.86 \%)$ & $4.823 \times 10^{5}(40.90 \%)$ \\
\hline 21 & 5.528 & $2.462 \times 10^{5}$ & $6.247(11.50 \%)$ & $4.002 \times 10^{5}(38.46 \%)$ \\
\hline 22 & 5.791 & $2.142 \times 10^{5}$ & $6.394(9.43 \%)$ & $3.359 \times 10^{5}(36.22 \%)$ \\
\hline 23 & 6.054 & $1.875 \times 10^{5}$ & $6.551(7.58 \%)$ & $2.848 \times 10^{5}(34.16 \%)$ \\
\hline 24 & 6.317 & $1.650 \times 10^{5}$ & $6.716(5.94 \%)$ & $2.437 \times 10^{5}(32.26 \%)$ \\
\hline 25 & 6.580 & $1.460 \times 10^{5}$ & $6.888(4.47 \%)$ & $2.102 \times 10^{5}(30.56 \%)$ \\
\hline
\end{tabular}

suggests (as in this case) that the dominant effect is to increase the modal stiffness. Assuming that the support legs are not a source of damping, it can be deduced that the frequency increase will also cause the Q-factor to increase. This argument is only valid if the thermoelastic mode shape is unchanged when support legs are attached. For very thin support legs modal mass dominates and the frequency is reduced. This behaviour is observed in the next section.

Similar characteristics are also observed when support legs are added to $8 \mathrm{~mm}$ diameter ring, see Table 4. Although, the large diameter thin rings give higher $Q_{T E D}$ values, the frequencies are in comparison quite low. Similar trends have been found when other leg configurations (e.g. as in Figures 2 (b) and 2 (c)) are used, but the results are not presented here. 
Table 4: Frequency and Q-factor of a $8 \mathrm{~mm}$ diameter rings with and without support legs and hub

\begin{tabular}{ccccc}
\hline \multirow{2}{*}{ Radial Thickness $b_{r}(\mu \mathrm{m})$} & \multicolumn{2}{c}{ Unsupported Ring } & \multicolumn{2}{c}{ Supported Ring } \\
\cline { 2 - 4 } & Frequency $(\mathrm{kHz})$ & $Q_{T E D}$ & Frequency $(\mathrm{kHz})$ & $Q_{T E D}$ \\
\hline 20 & 1.316 & $1.140 \times 10^{6}$ & $2.331(43.54 \%)$ & $3.432 \times 10^{6}(66.77 \%)$ \\
\hline 21 & 1.382 & $9.852 \times 10^{5}$ & $2.333(40.76 \%)$ & $2.791 \times 10^{6}(64.70 \%)$ \\
\hline 22 & 1.447 & $8.652 \times 10^{5}$ & $2.337(38.08 \%)$ & $2.293 \times 10^{6}(62.26 \%)$ \\
\hline 23 & 1.513 & $7.572 \times 10^{5}$ & $2.344(35.45 \%)$ & $1.900 \times 10^{6}(60.15 \%)$ \\
\hline 24 & 1.579 & $6.664 \times 10^{5}$ & $2.354(32.92 \%)$ & $1.589 \times 10^{6}(58.06 \%)$ \\
\hline 25 & 1.645 & $5.896 \times 10^{5}$ & $2.367(30.50 \%)$ & $1.340 \times 10^{6}(56.0 \%)$ \\
\hline
\end{tabular}

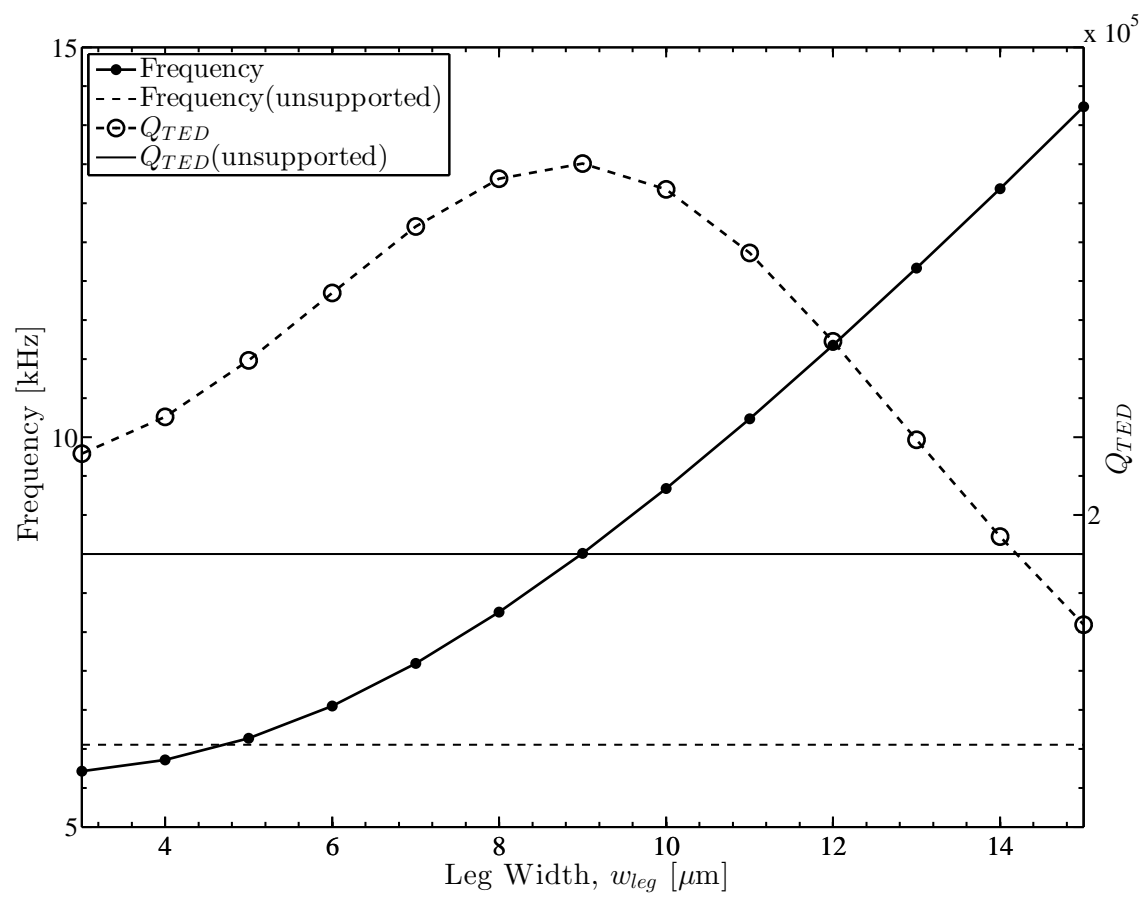

Figure 10: Effect of leg width on frequency and $Q_{T E D}$ for $4 \mathrm{~mm}-23 \mu \mathrm{m}$ rings.

\subsection{Effect of Leg Width on Frequency and $Q_{T E D}$}

Adding support legs was shown in the previous section to increase the frequency and $Q_{T E D}$ of the ring resonator. In this section, the influence of leg width on $Q_{T E D}$ is considered and this is achieved by considering two rings, with different diameters and thicknesses, and varying the leg width $w_{l e g}$. In addition the different leg configurations introduced earlier in Figure 2 are considered and for each of these cases the leg width is assumed constant and takes the same value in all sections of the leg. Figure 10 shows the frequency and $Q_{T E D}$ values for a ring of $4 \mathrm{~mm}$ diameter and $23 \mu \mathrm{m}$ radial thickness with z-shaped support legs (Figure 2(a)) having leg widths in the range $3-14 \mu \mathrm{m}$. The frequency increases non-linearly up to a certain $w_{l e g}$ value, after which it approximately increases linearly. For thin legs, e.g. $3 \mu \mathrm{m}$, the frequency 


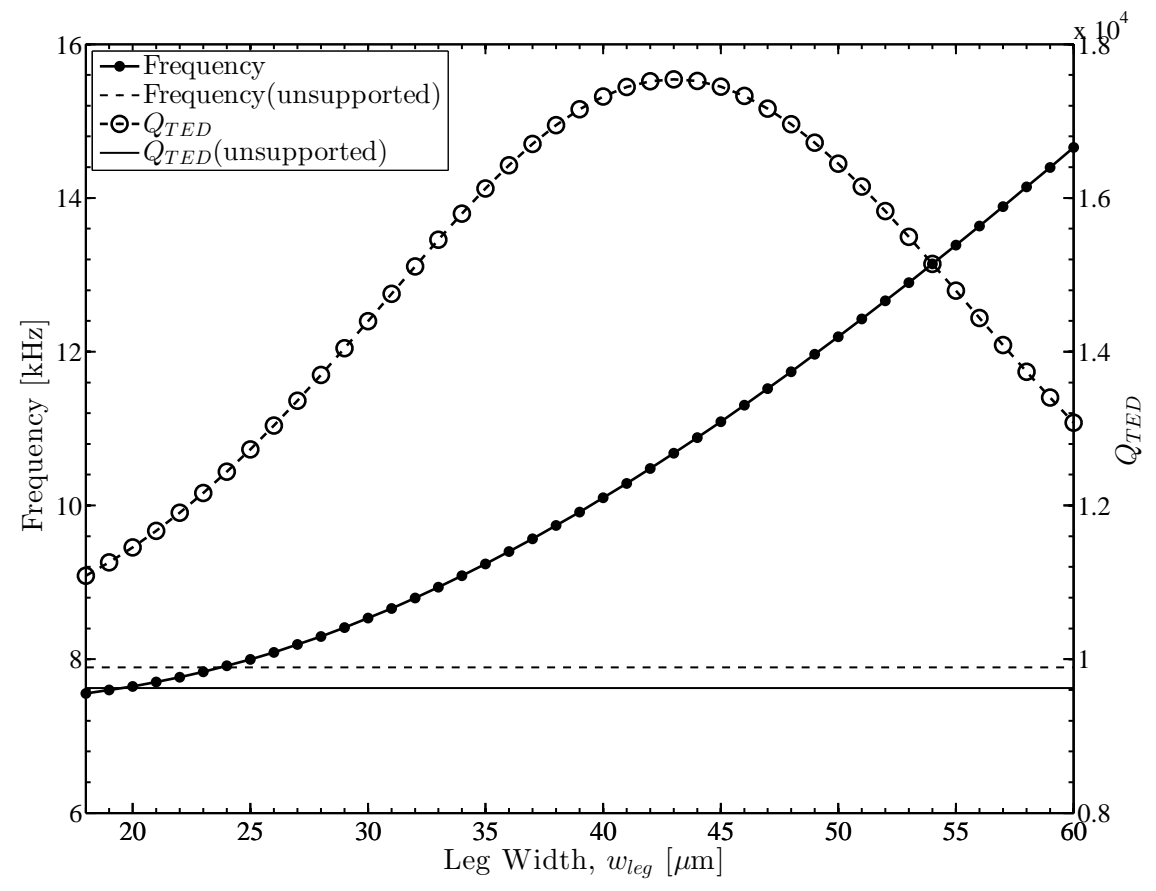

Figure 11: Effect of leg width on frequency and $Q_{T E D}$ for $8 \mathrm{~mm}-120 \mu \mathrm{m}$ rings.

(damped) of the supported ring is lower than the unsupported ring. This is due to the dominating effect of modal mass. As expected the natural frequency (damped) increases as the leg width increases due to increase in leg stiffness. However, the $Q_{T E D}$ value shows a different trend. The $Q_{T E D}$ increases gradually up to a peak value before decreasing with further increases in $w_{l e g}$. Although the frequency increases, $Q_{T E D}$ decreases significantly after reaching its peak value. This suggests that the support legs can have a significant influence on the overall damping, which is in contrast to the example considered in Section 5.1 where ring damping was observed to be dominant, based on a comparison with experiment. It is interesting to note that $Q_{T E D}$ reaches its peak at the $w_{l e g}$-value that coincides with the transition in frequency from increasing non-linearly to being in the linear range, see Figure 10 It is also worth noting that $Q_{T E D}$ of the supported ring could decrease compared to unsupported ring due to increased damping in thicker legs.

Similar characteristics can be observed for a large diameter thick ring with supports. Figure 11 shows the frequency and $Q_{T E D}$ values for a ring of $8 \mathrm{~mm}$ diameter and $120 \mu \mathrm{m}$ width for z-shaped legs having leg widths in the range $20 \mu \mathrm{m}$ to $60 \mu \mathrm{m}$. Similar to Figure $10 . Q_{T E D}$ increases to a peak value before decreasing with further increases in $w_{l e g}$. These results indicate that there are limits to the maximum value of $Q_{T E D}$ that can be achieved when support legs are included.

To further investigate this behaviour, semi-circular (see Figure 2(b)) support legs and a central hub are 


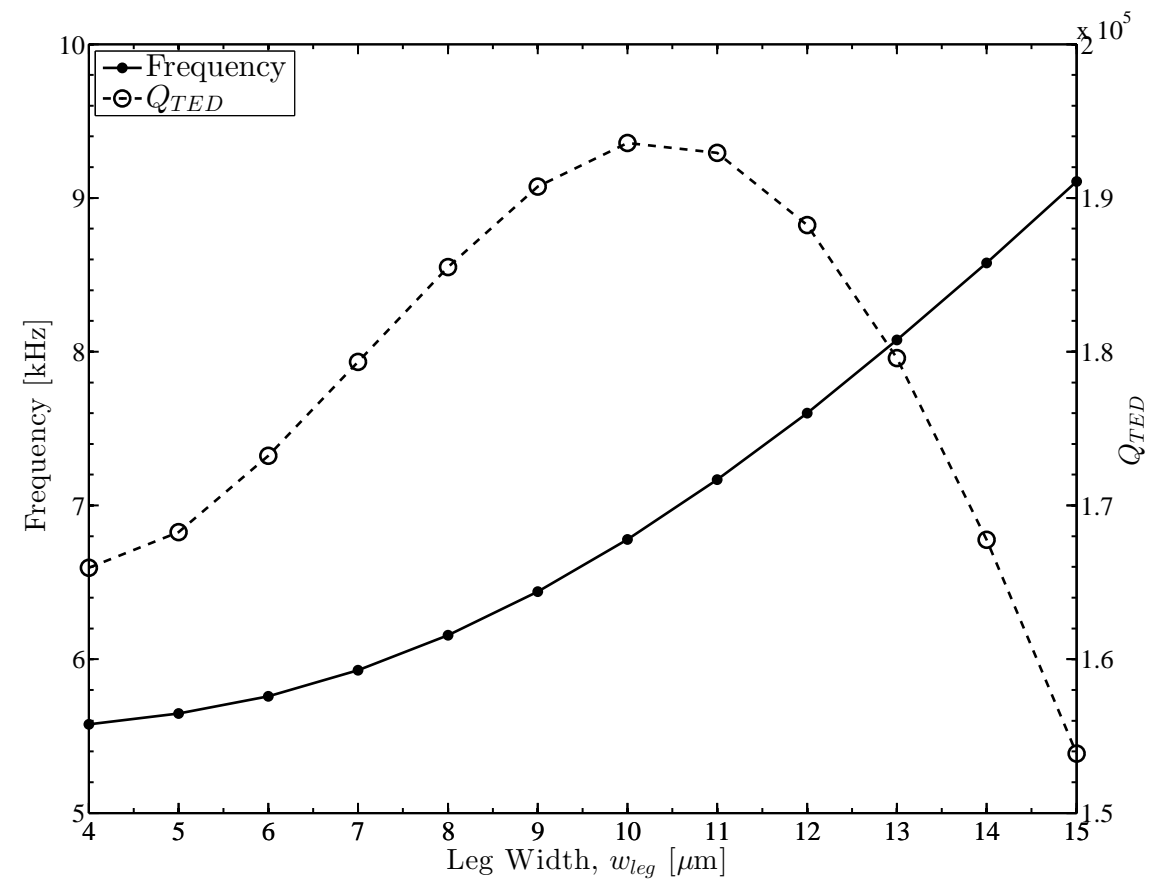

Figure 12: Effect of leg width (semi-circular support) on frequency and $Q_{T E D}$ for $4 \mathrm{~mm}-23 \mu \mathrm{m}$ rings.

implemented for a ring of $4 \mathrm{~mm}$ diameter and $23 \mu \mathrm{m}$ radial thickness. In Figure 12 , frequency and $Q_{T E D}$ are plotted as a function of $w_{l e g}$. The results show similar characteristics to those in Figures 10 and 11 . The $Q_{T E D}$ reaches a peak value before decreasing with $w_{l e g}$, and the frequency also increases approximately linearly. This trend is also apparent for $8 \mathrm{~mm}-120 \mu \mathrm{m}$ ring with semi-circular supports, see Figure 13 . Also, when supports outside of the rings are considered, (Figure 2(c)), similar behaviour for $Q_{T E D}$ and frequency is observed, see Figure 14. It can be inferred from the analyses that regardless of the support leg design, minimum damping occurs at an optimal leg width before damping starts to increase, i.e. $Q_{T E D}$ decreases.

\subsection{Influence of Leg Damping}

In the previous section it is shown that as the support leg width increases, $Q_{T E D}$ decreases after reaching a maximum even though the frequency increases monotonically. This behaviour suggests that as $w_{l e g}$ increases, the damping in the legs become significant.

This suggestion is investigated by re-considering the $4 \mathrm{~mm}-23 \mu \mathrm{m}$ ring with the support legs constrained so they have no TED this is achieved by modifying the coupling equation for the support legs. The results obtained for $Q_{T E D}$ when TED is limited to the ring only are compared with previous results including the legs as damping sources in Figure 15. For the case when TED is limited to the ring, $Q_{T E D}$ increases 


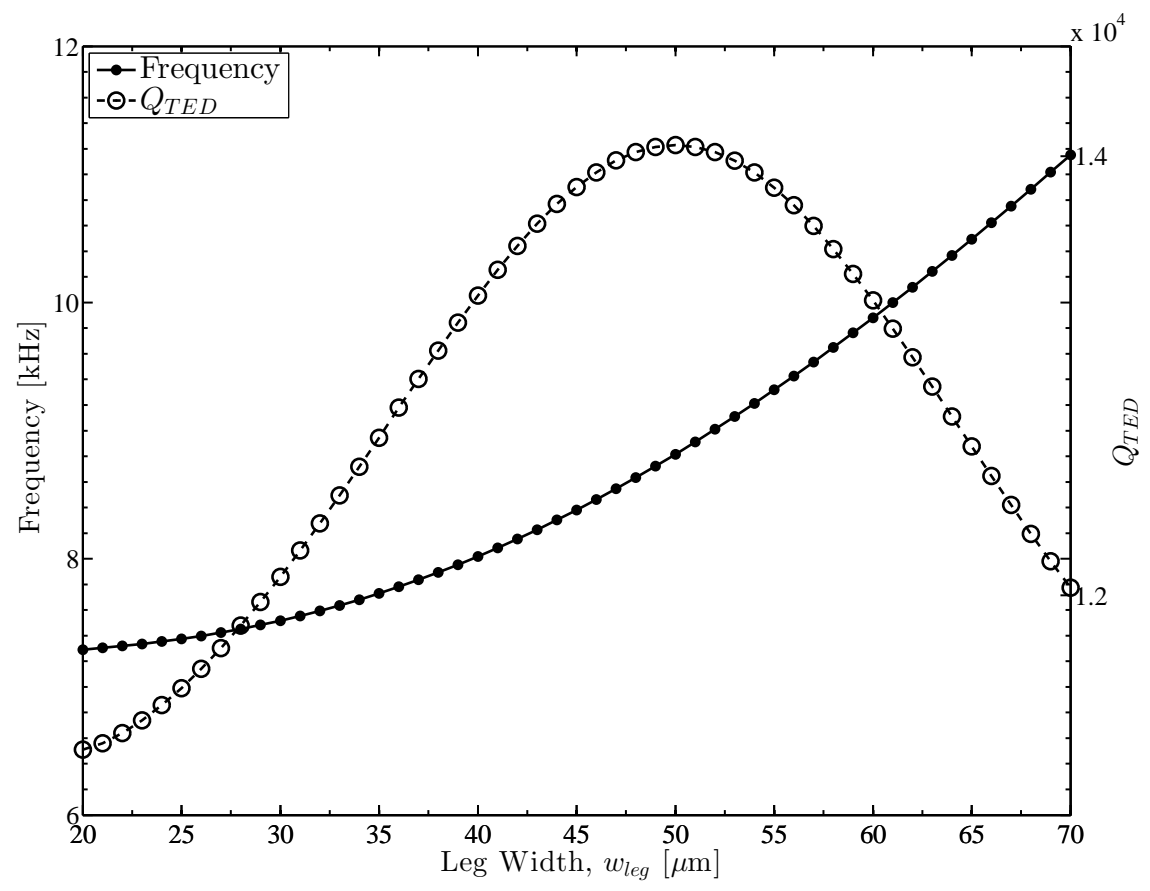

Figure 13: Effect of leg width (semi-circular support) on frequency and $Q_{T E D}$ for $8 \mathrm{~mm}-120 \mu \mathrm{m}$ rings.

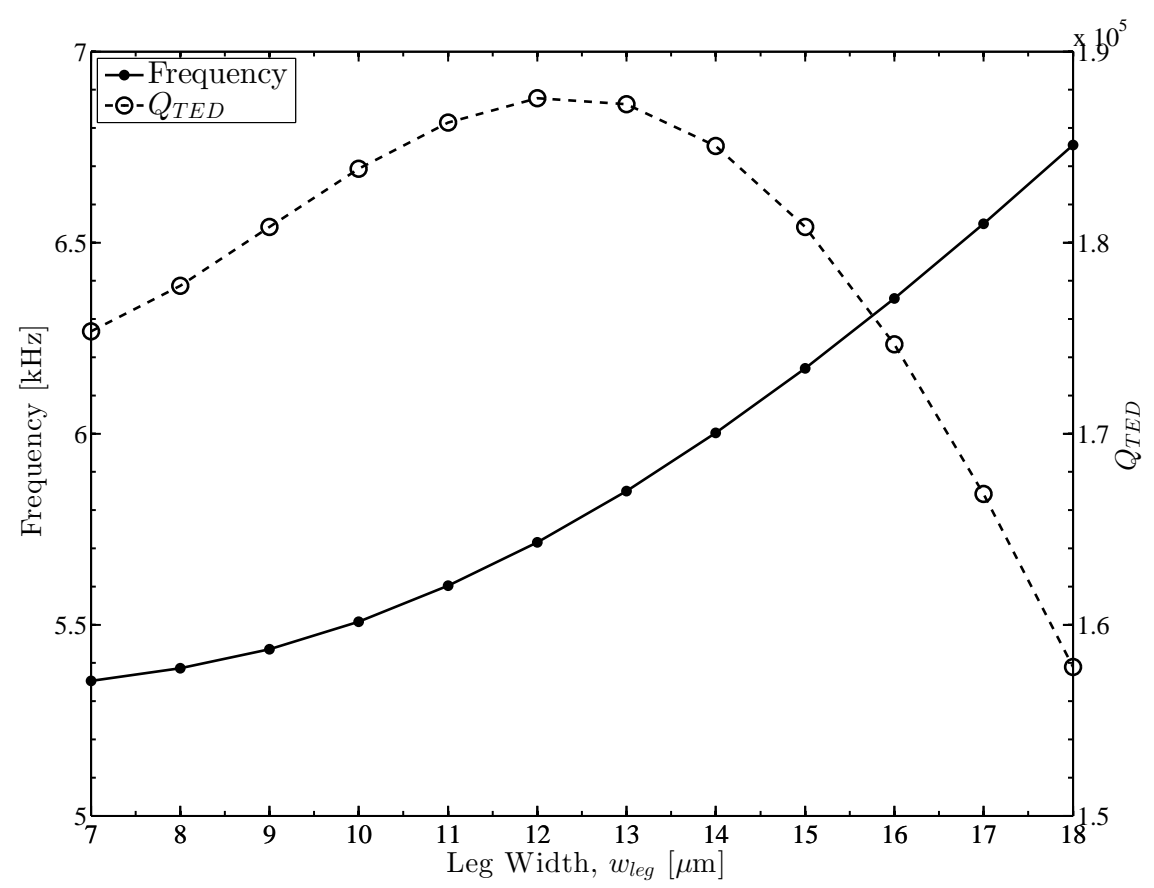

Figure 14: Effect of leg width (outer supports) on frequency and $Q_{T E D}$ for $4 \mathrm{~mm}-23 \mu \mathrm{m}$ rings. 


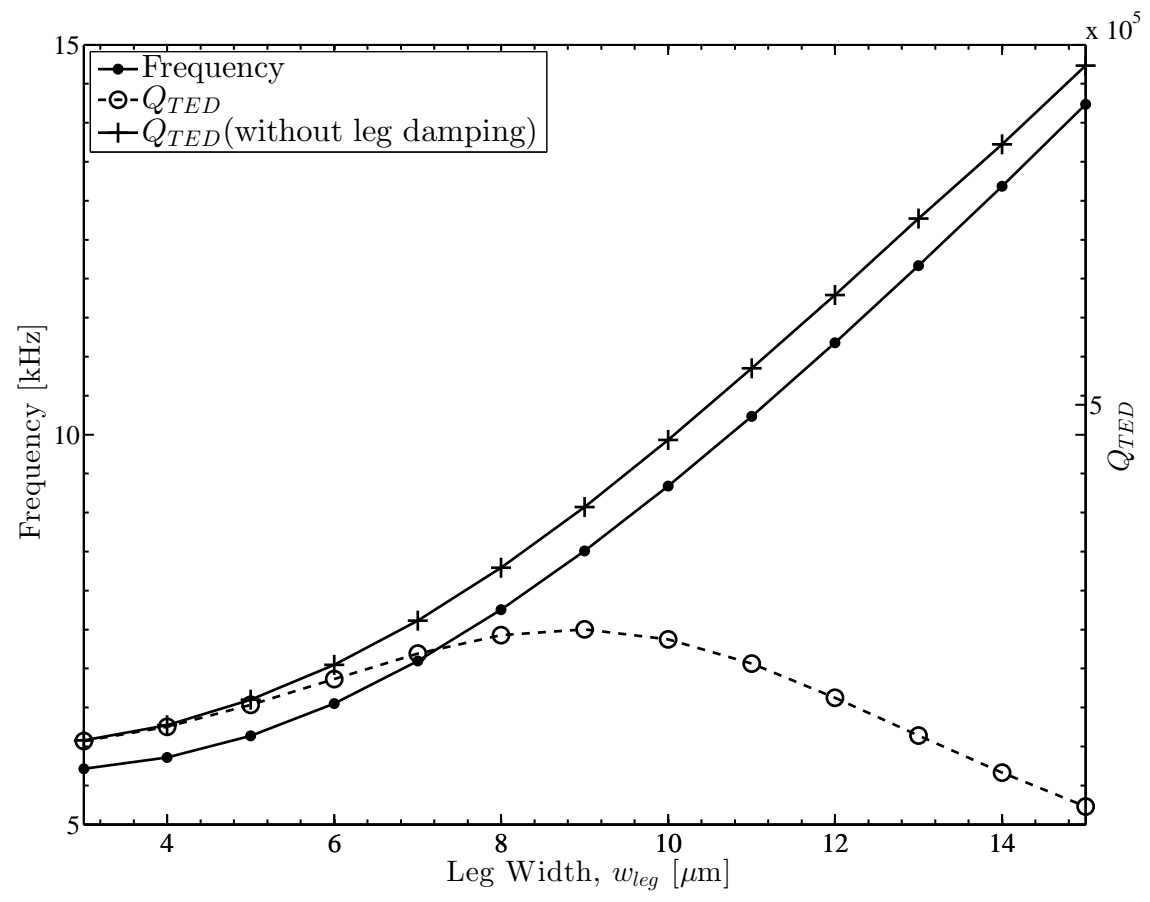

Figure 15: Influence of thermoelastic damping in support legs on $Q_{T E D}$ of a $4 \mathrm{~mm}-23 \mu \mathrm{m}$ ring.

monotonically as $w_{\text {leg }}$ increases. As in Section 6.1, this behaviour can be explained by noting that $Q_{T E D}$ increases as frequency increases provided that the thermoelastic mode shape is unchanged. When $w_{l e g}$ is small, the $Q_{T E D}$ values with and without leg damping are similar to each other, suggesting that leg damping is insignificant for small $w_{l e g}$ values. However, increasing $w_{l e g}$ introduces a significant amount of TED in the support legs which acts to reduce $Q_{T E D}$. For example, for a leg width of $14 \mu \mathrm{m}, Q_{T E D}$ is reduced by $\approx 72 \%$ due to the presence of leg damping.

\subsection{Damping in Different Leg Sections}

It is interesting to study how each part of the support leg contributes to the damping for supported rings with high damping. In this section only the z-shaped legs in Figure 2(a) are considered for brevity, and the damping in each section is achieved by dividing each 'z' shaped support leg into three sections, as shown in Figure 16 , and by evaluating the TED in each section.

Figure 17 shows results for the (high damping) $4 \mathrm{~mm}-23 \mu \mathrm{m}$ ring case with leg width $w_{l e g}=14 \mu \mathrm{m}$. It is worth mentioning that the results are interpreted in terms of loss factor which is the inverse of quality factor. The first column shows the overall loss factor $\left(\frac{1}{Q_{T E D}}\right)$ for the complete ring and support structure. The loss factor for the ring only is represented by $\frac{1}{Q_{\text {Ring }}}$ and $\frac{1}{Q_{P 1}}, \frac{1}{Q_{P 2}} \& \frac{1}{Q_{P 3}}$ are the individual loss factors 


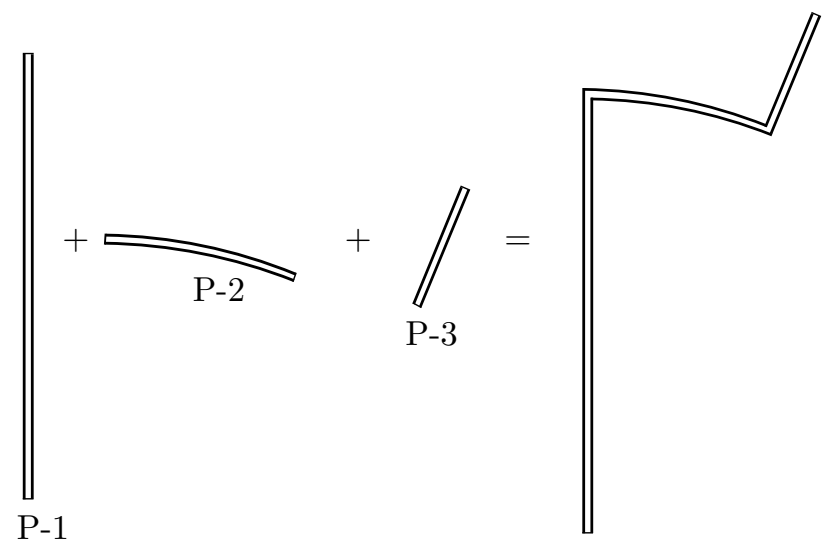

Figure 16: Different parts of support leg.

for the P-1, P-2 and P-3 sections, respectively. The overall loss factor $\left(\frac{1}{Q_{T E D}}\right)$ is the sum of all individual loss factors, such that:

$$
\frac{1}{Q_{T E D}}=\frac{1}{Q_{\text {Ring }}}+\frac{1}{Q_{P 1}}+\frac{1}{Q_{P 2}}+\frac{1}{Q_{P 3}}
$$

As expected the results indicate that leg damping is significant for the case considered. The results also indicate that damping in P-3 is higher than damping for the ring only. The TED in the ring only case accounts for $\approx 27.64 \%$ of the overall loss factor. Of the three different leg sections, P-3 has the highest damping and P-2 has the lowest energy. This is likely to be because this section (P-3) experiences higher levels of flexing, and consequently has a higher strain rate compared to the other sections.

\section{Thermoelastic Damping in Slotted Rings}

Candler et al. 21, 22, and Guo et al. 23, have shown that $Q_{T E D}$ of a beam resonator is influenced significantly by incorporating micro-machined slots in the beam. This section considers the influence of slots on various rings and provides an explanation for the damping behaviour of slotted rings.

\subsection{Effect of Slots on Rings}

To investigate the effect of slots, two different configurations of slots are considered, as shown in Figure 18 Figure 18 (a) shows a ring with single layer of slots arranged around the ring circumference on the neutral plane. Each slot has a width $w_{\text {slot }}$ of $6 \mu \mathrm{m}$ while the slot angular extent $\theta_{\text {slot }}$ is $3^{\circ}$ and the angular gap between neighbouring slots $\theta_{\text {slot-gap }}$ is of $5^{\circ}$. Figure 18 (b) shows a ring with a double layer of slots. In this case the slots are positioned at equal distance $(2 \mu \mathrm{m})$ from the neutral plane and each slot layer has $w_{\text {slot }}=2 \mu \mathrm{m}, \theta_{\text {slot }}=2^{\circ}$ and $\theta_{\text {slot-gap }}=1^{\circ}$. From the previous results obtained, it is known that a ring 


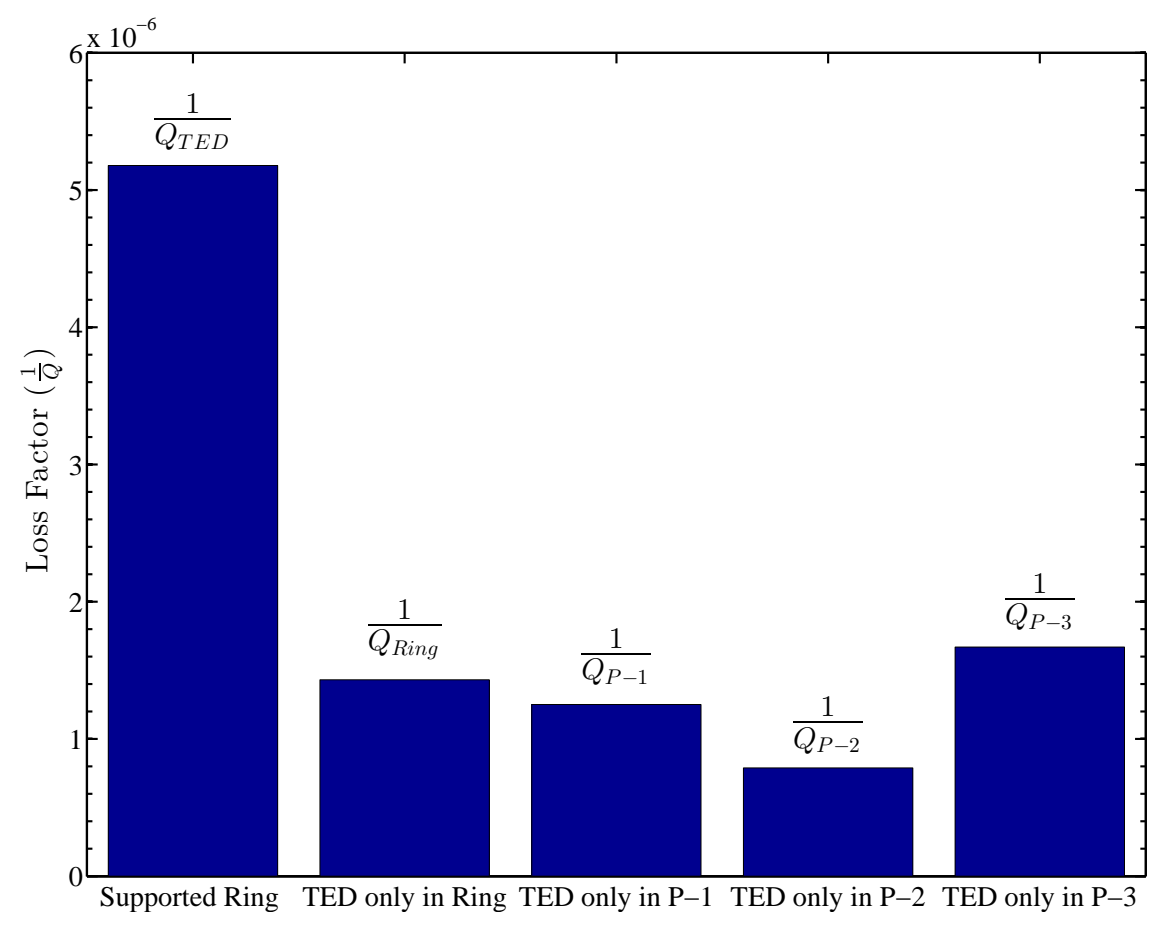

Figure 17: Loss factor $\left(\frac{1}{Q}\right)$ in different leg sections of $4 \mathrm{~mm}-23 \mu \mathrm{m}$ ring with $w_{l e g}=14 \mu \mathrm{m}$.

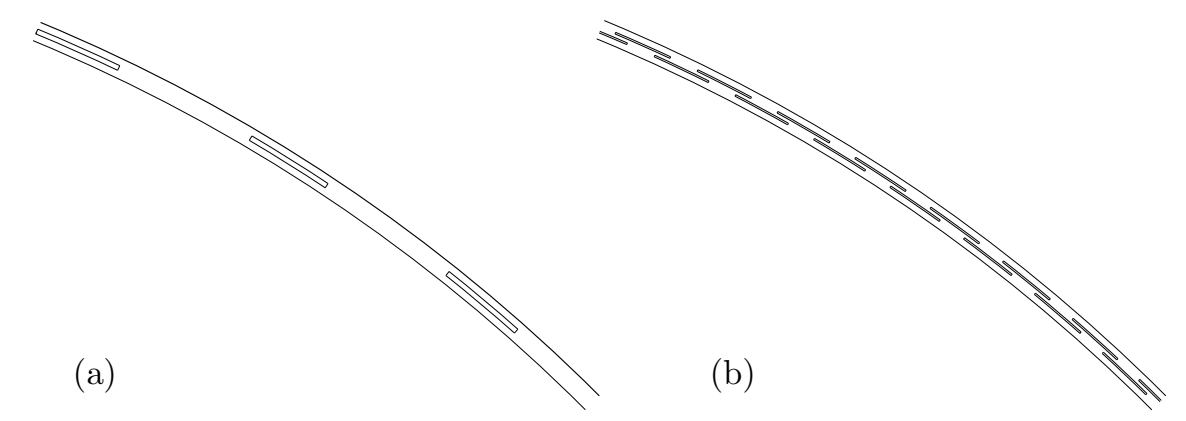

Figure 18: (a) Single and (b) double layer slots equally positioned from the neutral plane. 
with $8 \mathrm{~mm}$ diameter and $120 \mu \mathrm{m}$ radial thickness has relatively low $Q_{T E D}$ value due to operating near the Debye peak region. The two slot configurations are used on this ring, and results obtained using numerical simulations for unslotted and slotted cases are shown in Table 5. It is clear that the presence of micromachined slots increases $Q_{T E D}$ in this case. Slots work by disrupting the heat flow due to temperature

Table 5: Frequency and $Q_{T E D}$ of $8 \mathrm{~mm}-120 \mu \mathrm{m}$ ring with single and double layer slots

\begin{tabular}{cccccc}
\hline \multirow{2}{*}{ Unslotted Ring } & \multicolumn{4}{c}{ Slots Included } \\
\cline { 2 - 5 } & \multicolumn{2}{c}{ Single Layer } & \multicolumn{2}{c}{ Double Layer } \\
\hline Frequency $(\mathrm{kHz})$ & $Q_{T E D}$ & Frequency $(\mathrm{kHz})$ & $Q_{T E D}$ & Frequency $(\mathrm{kHz})$ & $Q_{T E D}$ \\
\hline 7.895 & 9624 & 7.959 & 13347 & 7.455 & 61162 \\
\hline
\end{tabular}

gradients formed in different parts of the ring, causing $Q_{T E D}$ to increase, more significantly for the double layer slots. Of course, $Q_{T E D}$ of slotted rings is influenced by various factors like: number of slots, location of slots, slot sizes and gaps, but this is not the purpose of this paper and these factors are not directly discussed here.

To investigate the effect of slots on high-Q ring resonators, $4 \mathrm{~mm}$ diameter rings with radial thicknesses in the range $20-25 \mu \mathrm{m}$ are selected based on results shown in Figure 7. These rings operate in the isothermal region of the Debye curve, and so have high-Q values. Table 6 shows numerically simulated results for the frequency and $Q_{T E D}$ for rings with single and double layer slots, and no support legs. For the rings considered including slots causes $Q_{T E D}$ to decrease significantly, indicating that the damping has been increased - this is in contrast to the results in Table 5 where the damping was reduced. Table 7 shows similar information to Table 6 but for a $2 \mathrm{~mm}$ diameter ring with radial thicknesses in the range $140-160 \mu \mathrm{m}$, which operate in the adiabatic region. Again it is found that including slots causes the damping to increase, however for the selected slots the decrease in $Q_{T E D}$ is not as high as the isothermal ring mentioned above.

In summary the results presented suggest that slots can improve $Q_{T E D}$ for a ring having low Q, i.e. operating near the Debye peak, but slots do not improve $Q_{T E D}$ for the high-Q rings that operate in isothermal or adiabatic region.

\subsection{Damping Behaviour in Slotted Rings}

Candler et al. 21] mention in their work that the addition of slots can decrease the Q-factor, depending on the specific geometry and frequency of a thermoelastically limited beam resonator. This behaviour has also been observed in 22, 24, for flexural beam resonators. In the previous section it was found that $Q_{T E D}$ reduces in some cases when slots are added. Due to the complexity added by the slots to the geometry of the ring, Zener's theory based on single thermal mode is not sufficient to explain this behaviour. Candler 
Table 6: Frequency and $Q_{T E D}$ of $4 \mathrm{~mm}$ ring with single and double layer slots

\begin{tabular}{ccccccc}
\hline Radial Thickness & \multicolumn{2}{c}{ Unslotted Ring } & \multicolumn{4}{c}{ Slots Included } \\
\cline { 2 - 6 }$b_{r}$ & \multicolumn{2}{c}{ Single Layer } & \multicolumn{2}{c}{ Double Layer } \\
\cline { 2 - 6 }$(\mu \mathrm{m})$ & Freq. $(\mathrm{kHz})$ & $Q_{T E D}$ & Freq. $(\mathrm{kHz})$ & $Q_{T E D}$ & Freq. $(\mathrm{kHz})$ & $Q_{T E D}$ \\
\hline 20 & 5.264 & $2.850 \times 10^{5}$ & 5.531 & $3.086 \times 10^{4}$ & 5.503 & $1.308 \times 10^{4}$ \\
\hline 21 & 5.528 & $2.462 \times 10^{5}$ & 5.797 & $2.977 \times 10^{4}$ & 5.773 & $1.260 \times 10^{4}$ \\
\hline 22 & 5.791 & $2.142 \times 10^{5}$ & 6.063 & $2.879 \times 10^{4}$ & 6.041 & $1.228 \times 10^{4}$ \\
\hline 23 & 6.054 & $1.875 \times 10^{5}$ & 6.327 & $2.789 \times 10^{4}$ & 6.309 & $1.210 \times 10^{4}$ \\
\hline 24 & 6.317 & $1.650 \times 10^{5}$ & 6.592 & $2.708 \times 10^{4}$ & 6.575 & $1.202 \times 10^{4}$ \\
\hline 25 & 6.580 & $1.460 \times 10^{5}$ & 6.857 & $2.633 \times 10^{4}$ & 6.840 & $1.204 \times 10^{4}$ \\
\hline
\end{tabular}

Table 7: Frequency and $Q_{T E D}$ of $2 \mathrm{~mm}$ ring with single and double layer slots

\begin{tabular}{|c|c|c|c|c|c|c|}
\hline \multirow{3}{*}{$\begin{array}{c}\text { Radial Thickness } \\
b_{r} \\
(\mu \mathrm{m}) \\
\end{array}$} & \multirow{2}{*}{\multicolumn{2}{|c|}{ Unslotted Ring }} & \multicolumn{4}{|c|}{ Slots Included } \\
\hline & & & \multicolumn{2}{|c|}{ Single Layer } & \multicolumn{2}{|c|}{ Double Layer } \\
\hline & Freq. (kHz) & $Q_{T E D}$ & Freq. $(\mathrm{kHz})$ & $Q_{T E D}$ & Freq. (kHz) & $Q_{T E D}$ \\
\hline 140 & 146.26 & $9.966 \times 10^{4}$ & 146.94 & $9.957 \times 10^{4}$ & 144.31 & $9.586 \times 10^{4}$ \\
\hline 145 & 151.40 & $1.095 \times 10^{5}$ & 152.05 & $1.090 \times 10^{5}$ & 149.22 & $1.031 \times 10^{5}$ \\
\hline 150 & 156.52 & $1.197 \times 10^{5}$ & 157.15 & $1.191 \times 10^{5}$ & 154.11 & $1.105 \times 10^{5}$ \\
\hline 155 & 161.64 & $1.314 \times 10^{5}$ & 162.22 & $1.294 \times 10^{5}$ & 158.98 & $1.178 \times 10^{5}$ \\
\hline 160 & 166.75 & $1.439 \times 10^{5}$ & 167.30 & $1.405 \times 10^{5}$ & 163.84 & $1.252 \times 10^{5}$ \\
\hline
\end{tabular}

et al. 22] proposed a rigorous explanation based on coupling of thermal and mechanical modes. The same explanation can be applied to the case of slotted high-Q rings. Due to thermoelastic coupling, several thermal modes can couple with the mechanical mode of interest. For an unslotted ring, it is a valid approximation (based on Zener's theory) that a single thermal mode (usually the first mode) is strongly coupled to the mechanical mode, and $Q_{T E D}$ is dominated by the energy dissipation from that mode. However, the addition of slots causes multiple thermal modes to be coupled to the mechanical mode. The initial thermal mode of an unslotted high-Q ring resonator is fully coupled to the mechanical mode and has very low energy dissipation if it is operating in the isothermal or adiabatic regime. Adding slots to the ring couples the mechanical mode to higher frequency thermal modes. This reduces the coupling effect with the initial thermal mode. The partially coupled higher thermal modes also have energy dissipation. This dissipation is significantly higher than the energy dissipated by the initial thermal mode which becomes partially coupled when slots are added, leading to a reduction in $Q_{T E D}$. Figure 19 shows the change in temperature distribution due to the addition of slots into a ring operating in the isothermal regime. A similar explanation is valid for the case where $Q_{T E D}$ of a ring resonator, operating near the Debye peak, increases by the addition of slots. In this case, the initial thermal mode has higher energy dissipation, and incorporating slots reduces the coupling of this mode with the mechanical mode. Also, the energy dissipation from the partially coupled higher frequency thermal modes is such that it does not decrease $Q_{T E D}$. In summary, the addition of slots 


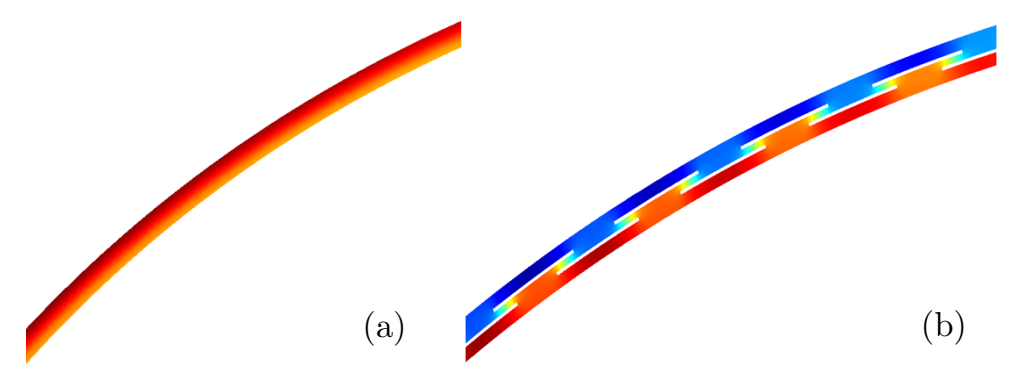

Figure 19: Temperature distribution in (a) unslotted and (b) slotted high-Q rings. Red and blue indicates higher and lower temperatures, respectively.

introduces a complex coupling of thermal modes and the mechanical mode of interest which can increase or decrease $Q_{T E D}$ depending on whether the ring is operating near or away (isothermal/adiabatic regime) from the Debye peak.

\section{Conclusions}

Numerical finite element based simulations and available analytical solutions have been used to predict thermoelastic damping (TED) in ring resonators. The key purpose of the work is to present a parameter study to investigate the influence of bulk geometry changes in the ring and support structure and ring slot enhancements on TED, providing explanations for the observed behaviour where possible. The initial step taken was to understand the damping behaviour of unsupported rings. The TED behaviour of unsupported rings is adequately explained by Zener's theory, and as such it is possible to achieve high-Q rings by operating in either the isothermal or adiabatic regions. In practice this can be achieved by using either large diameter thin rings or small diameter thick rings. However, large diameter thin rings have lower frequencies than small diameter thick rings, and this means that there is a trade-off between frequency and $Q$ that needs to be addressed when designing high-Q resonators for rate sensor applications. Adding support legs to high-Q rings tends to increase Q-factors compared to unsupported rings, but depending on the support leg design and ring size it could decrease also. However, as the width of the supporting legs increases, the Q-factor increases to a maximum before decreasing, suggesting that there is an optimum leg width at which maximum $Q$ is achieved. This behaviour is attributed to TED in the support legs which is dominant compared to the damping in the ring. The effect of incorporating slots around the ring circumference was also investigated for high-Q unsupported rings, and it was found that slots can decrease $Q$ (increase damping) by reducing coupling between thermal modes and the prevailing mechanical mode. 


\section{Acknowledgement}

The authors gratefully acknowledge the support for this work provided by UTC Aerospace Systems, formerly Atlantic Inertial Systems (AIS). The views expressed in the paper are those of the authors and do not necessarily reflect the views of UTC Aerospace Systems.

\section{References}

[1] N. Yazdi, F. Ayazi, K. Najafi, Micromachined inertial sensors, Proceedings of the IEEE 86 (8) (1998) 1640-1659. doi: $10.1109 / 5.704269$

[2] M. Weinberg, R. Candler, S. Chandorkar, J. Varsanik, T. Kenny, A. Duwel, Energy loss in MEMS resonators and the impact on inertial and RF devices, in: Solid-State Sensors, Actuators and Microsystems Conference, 2009. TRANSDUCERS 2009. International, 2009, pp. 688-695. doi:10.1109/SENSOR.2009.5285418

[3] Y.-B. Yi, A. Rahafrooz, S. Pourkamali, Modeling and testing of the collective effects of thermoelastic and fluid damping on silicon MEMS resonators, Journal of Micro/Nanolithography, MEMS, and MOEMS 8 (2) (2009) 023010-023010-7. doi: $10.1117 / 1.3129830$

[4] Z. Hao, A. Erbil, F. Ayazi, An analytical model for support loss in micromachined beam resonators with in-plane flexural vibrations, Sensors and Actuators A: Physical 109 (12) (2003) 156 - 164. doi:10.1016/j.sna.2003.09.037

[5] J. A. Judge, D. M. Photiadis, J. F. Vignola, B. H. Houston, J. Jarzynski, Attachment loss of micromechanical and nanomechanical resonators in the limits of thick and thin support structures, Journal of Applied Physics 101 (1) (2007) 013521-11. doi:10.1063/1.2401271

[6] B. Chouvion, S. McWilliam, C. H. J. Fox, A. A. Popov, Review and comparison of different support loss models for micro-electro-mechanical systems resonators undergoing in-plane vibration, Proceedings of the Institution of Mechanical Engineers, Part C: Journal of Mechanical Engineering Science 226 (1) (2012) 283-295. doi:10.1177/0954406211413351

[7] C. Zener, Internal friction in solids. i. theory of internal friction in reeds, Physical Review 52 (1937) 230-235. doi: 10.1103/PhysRev.52.230

[8] C. Zener, Internal friction in solids ii. general theory of thermoelastic internal friction, Physical Review 53 (1938) 90-99. doi:10.1103/PhysRev.53.90

[9] R. Lifshitz, M. L. Roukes, Thermoelastic damping in micro- and nanomechanical systems, Phyical Review B 61 (2000) 5600-5609. doi:10.1103/PhysRevB.61.5600.

[10] H. Sheng, H. Li, P. Lu, H. Xu, Free vibration analysis for micro-structures used in MEMS considering surface effects, Journal of Sound and Vibration 329 (2) (2010) 236 - 246. doi:10.1016/j.jsv.2009.08.035.

[11] H. Campanella, Acoustic Wave and Electromechanical Resonators: Concept to Key Applications, Artech House, Massachusetts, 2010 .

[12] D. S. Bindel, S. Govindjee, Elastic PMLs for resonator anchor loss simulation, International Journal for Numerical Methods in Engineering 64 (6) (2005) 789-818. doi:10.1002/nme.1394

[13] Z. Hao, F. Ayazi, Support loss in the radial bulk-mode vibrations of center-supported micromechanical disk resonators, Sensors and Actuators A: Physical 134 (2) (2007) 582-593. doi:10.1016/j.sna.2006.05.020 
[14] T. Roszhart, The effect of thermoelastic internal friction on the Q of micromachined silicon resonators, in: Solid-State Sensor and Actuator Workshop, 1990. 4th Technical Digest., IEEE, 1990, pp. 13-16. doi:10.1109/SOLSEN.1990.109810

[15] A. Duwel, J. Gorman, M. Weinstein, J. Borenstein, P. Ward, Experimental study of thermoelastic damping in MEMS gyros, Sensors and Actuators A: Physical 103 (12) (2003) 70 - 75. doi:10.1016/S0924-4247(02)00318-7

[16] F. Ayazi, K. Najafi, A HARPSS polysilicon vibrating ring gyroscope, Journal of Microelectromechanical Systems 10 (2) (2001) 169-179. doi:10.1109/84.925732

[17] H. Najar, A. Heidari, M.-L. Chan, H.-A. Yang, L. Lin, D. G. Cahill, D. A. Horsley, Microcrystalline diamond micromechanical resonators with quality factor limited by thermoelastic damping, Applied Physics Letters 102 (7). doi:10.1063/1.4793234

[18] S. J. Wong, C. H. J. Fox, S. McWilliam, C. P. Fell, R. Eley, A preliminary investigation of thermo-elastic damping in silicon rings, Journal of Micromechanics and Microengineering 14 (9) (2004) S108-S113. doi:10.1088/0960-1317/14/9/019

[19] S. J. Wong, C. H. J. Fox, S. McWilliam, Thermoelastic damping of the in-plane vibration of thin silicon rings, Journal of Sound and Vibration 293 (1-2) (2006) 266-285. doi:10.1016/j.jsv.2005.09.037

[20] Z. Hao, F. Ayazi, Thermoelastic damping in flexural-mode ring gyroscopes, in: ASME 2005 International Mechanical Engineering Congress and Exposition, 2005, pp. 335-343. doi:10.1115/IMECE2005-79965

[21] R. Candler, M. Hopcroft, C. Low, S. Chandorkar, B. Kim, M. Varghese, A. Duwel, T. Kenny, Impact of slot location on thermoelastic dissipation in micromechanical resonators, in: Solid-State Sensors, Actuators and Microsystems, 2005. Digest of Technical Papers. TRANSDUCERS '05. The 13th International Conference on, Vol. 1, 2005, pp. 597-600. doi:10.1109/SENSOR.2005.1496488

[22] R. Candler, A. Duwel, M. Varghese, S. A. Chandorkar, M. Hopcroft, W.-T. Park, B. Kim, G. Yama, A. Partridge, M. Lutz, T. Kenny, Impact of geometry on thermoelastic dissipation in micromechanical resonant beams, Journal of Microelectromechanical Systems 15 (4) (2006) 927-934. doi:10.1109/JMEMS.2006.879374

[23] X. Guo, Y.-B. Yi, S. Pourkamali, A finite element analysis of thermoelastic damping in vented MEMS beam resonators, International Journal of Mechanical Sciences 74 (0) (2013) 73-82. doi:10.1016/j.ijmecsci.2013.04.013

[24] A. Duwel, R. Candler, T. Kenny, M. Varghese, Engineering MEMS resonators with low thermoelastic damping, Journal of Microelectromechanical Systems 15 (6) (2006) 1437-1445. doi:10.1109/JMEMS.2006.883573

[25] COMSOL Multiphysics $4.2 \mathrm{a}$ is a product of COMSOL Ltd. URL http://www.uk.comsol.com

[26] C. H. J. Fox, Vibrating cylinder rate gyro: theory of operation and error analysis, in: Proceedings of the DGON Symposium on Gyro Technology, Stuttgart, 1988.

[27] V. Kinra, K. Milligan, A second-law analysis of thermoelastic damping, Journal of Applied Mechanics 61 (1) (1994) 71-76. doi:10.1115/1.2901424

[28] M. Blanter, I. Golovin, H. Neuhäuser, H. Sinning, Internal Friction in Metallic Materials: A Handbook, Springer Series in Materials Science, Springer, Berlin, 2007.

[29] N. Noda, R. Hetnarski, Y. Tanigawa, Thermal Stresses, 2nd Edition, Taylor \& Francis, New York, 2003.

[30] G. He, K. Najafi, A single-crystal silicon vibrating ring gyroscope, in: The Fifteenth IEEE International Conference on Micro Electro Mechanical Systems, 2002, pp. 718-721. doi:10.1109/MEMSYS.2002.984371

[31] R. Blevins, Formulas for Natural Frequency and Mode Shape, Krieger Publishing Company, Florida, 1979.

[32] J. Liewald, B. Kuhlmann, T. Balslink, Y. Manoli, A 100 kHz vibratory MEMS rate gyroscope with experimental verification 
of system model's frequency scaling, in: IEEE 25th International Conference on Micro Electro Mechanical Systems (MEMS), 2012, pp. 492-495. doi:10.1109/MEMSYS.2012.6170245 